ARTICLE

\title{
Structure of HIV-1 Vpr in complex with the human nucleotide excision repair protein hHR23A
}

In-Ja L. Byeon 1,2,5, Guillermo Calero (1) 1,2,5凶 , Ying Wu,2, Chang H. Byeon (1) 2, Jinwon Jung (1) 2,3, Maria DeLucia ${ }^{1,2}$, Xiaohong Zhou (10 ${ }^{1,2}$, Simon Weiss ${ }^{2}$, Jinwoo Ahn (i) ${ }^{1,2}$, Caili Hao ${ }^{1,4}$, Jacek Skowronski ${ }^{1,4} \&$ Angela M. Gronenborn (10) 1,2凶

HIV-1 Vpr is a prototypic member of a large family of structurally related lentiviral virulence factors that antagonize various aspects of innate antiviral immunity. It subverts host cell DNA repair and protein degradation machineries by binding and inhibiting specific post-replication repair enzymes, linking them via the DCAF1 substrate adaptor to the Cullin 4 RING E3 ligase (CRL4DCAF1). HIV-1 Vpr also binds to the multi-domain protein hHR23A, which interacts with the nucleotide excision repair protein XPC and shuttles ubiquitinated proteins to the proteasome. Here, we report the atomic resolution structure of $\mathrm{Vpr}$ in complex with the C-terminal half of hHR23A, containing the XPC-binding (XPCB) and ubiquitin-associated (UBA2) domains. The XPCB and UBA2 domains bind to different sides of Vpr's 3-helixbundle structure, with UBA2 interacting with the $\alpha 2$ and $\alpha 3$ helices of Vpr, while the XPCB domain contacts the opposite side of Vpr's $\alpha 3$ helix. The structure as well as biochemical results reveal that hHR23A and DCAF1 use overlapping binding surfaces on Vpr, even though the two proteins exhibit entirely different three-dimensional structures. Our findings show that $\mathrm{Vpr}$ independently targets hHR23A- and DCAF1- dependent pathways and highlight HIV-1 Vpr as a versatile module that interferes with DNA repair and protein degradation pathways.

\footnotetext{
${ }^{1}$ Pittsburgh Center for HIV Protein Interactions, University of Pittsburgh School of Medicine, Pittsburgh, PA, USA. ${ }^{2}$ Department of Structural Biology, University of Pittsburgh School of Medicine, Pittsburgh, PA, USA. ${ }^{3}$ Present address: ABL Bio Inc., 16, Daewangpangyo-ro 712 beon-gil, Bundang-gu, Seongnam-si, Gyeonggi-do 13488, Republic of Korea. ${ }^{4}$ Present address: Department of Molecular Biology and Microbiology, Case Western Reserve School of Medicine, Cleveland, OH, USA. ${ }^{5}$ Theses authors contributed equally: In-Ja L Byeon, Guillermo Calero. ${ }^{\bigotimes_{e}}$ mail: guc9@pitt.edu; amg100@pitt.edu
} 
$\mathrm{V}$ pr is one of the four HIV-1 accessory virulence factors, Vif, Vpr, Vpu, and Nef, that antagonize innate mechanisms and intrinsic cellular pathways that interfere with effective viral replication ${ }^{1-7}$. Closely structurally related orthologues and paralogues of HIV-1 Vpr are found in HIV-2 and essentially all primate lentiviruses ${ }^{8-10}$. While our knowledge of how Vpr promotes HIV-1 replication and pathogenesis is clearly incomplete, one well-established mechanism involves hijacking of the CRL4 E3 ubiquitin ligase and its DCAF1 substrate receptor (CRL4 ${ }^{\text {DCAF1 }}$ ) for depletion of cellular proteins that directly or indirectly target viral components. In particular, $\mathrm{Vpr}$ binds to DCAF1 and mediates loading of different protein substrates onto this E3 ligase, leading to their polyubiquitination and proteasome-dependent degradation ${ }^{11-13}$. Intriguingly, among these substrates are proteins involved in post-replication DNA repair and chromatin metabolism, including the base excision repair (BER) protein Uracil-DNA glycosylase (UNG2) ${ }^{14,15}$, the double strand break repair and Holliday junction resolvase MUS81-EME1 16,17, the helicase-like transcription factor (HLTF) that plays a role in the repair of stalled replication forks ${ }^{18-20}$, Exo1, a nuclease involved in double strand break repair ${ }^{21}$, and TET2, a methylcytosine dioxygenase that is a potent epigenetic regulator of gene expression in hematopoietic cells ${ }^{22}$. The structural basis of E3 ligase targeting by Vpr was elucidated for the BER protein UNG2: the substrate receptor DCAF1 engages helix $\alpha 3$ and, on the opposing surface of Vpr, a loop between helices a2 and $\alpha 3$ inserts into the active site of UNG2, thereby interfering with DNA binding and catalysis ${ }^{23}$. Significantly, UNG2, Exo1, HLTF and TET2 were shown to interfere with HIV-1 infection, supporting the notion that $\mathrm{Vpr}$ antagonizes specific postreplication DNA repair proteins that restrict HIV-1, thereby facilitating HIV-1 replication $4,21,22,24$.

HIV-1 Vpr also binds tightly to hHR23A ${ }^{25-27}$, the human homolog of yeast Rad23, which not only partakes in nucleotide excision repair (NER) but also plays important roles in various other aspects of cellular metabolism, beyond DNA repair ${ }^{28}$. hHR23A and its hHR23B isoform are modular and comprise four independently folded domains, joined by three flexible linkers. In particular, they possess an N-terminal ubiquitin-like domain (UBL), a central ubiquitin-associated domain (UBA1), followed by a RAD4/XPC (xeroderma pigmentosum group $\mathrm{C}$ ) binding domain (RBD/XPCB) and a C-terminal ubiquitin-associated domain (UBA2). hHR23 proteins participate in NER by forming a complex with the XPC protein, mediated by the XPCB domain, and play a role in the initial recognition of damaged sites in $\mathrm{DNA}^{29}$. In addition, the hHR23B-XPC complex was shown to function as a co-activator controlling particular transcription programs $^{30-32}$. The UBL domain mediates 26S proteasome binding by engaging the Rpn 1 and Rpn 10 subunits $^{33,34}$, while the UBA domains capture polyubiquitin-conjugated target proteins, especially Lys-48-conjugated chains ${ }^{35}$.

Previously, we and others demonstrated that $\mathrm{Vpr}$, which comprises a three-helix bundle with flexible $\mathrm{N}$ - and C-terminal tails ${ }^{23,36}$, binds the C-terminal half of hHR23A, contacting the $\mathrm{UBA}^{25}$ and the $\mathrm{XPCB}^{37}$ domains. Interestingly, in contrast to other DNA repair proteins that are targeted by Vpr, hHR23A does not become ubiquitinated in a Vpr-dependent manner and is not depleted in cells expressing HIV-1 $\mathrm{Vpr}^{21}$, suggesting

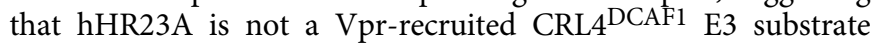
and that the Vpr-hHR23A complex likely presents a distinct architecture.

Here, we report the high-resolution NMR structure of Vpr, complexed with the C-terminal half of hHR23A, containing the $\mathrm{XPCB}$ and UBA2 domains, and a $2.2 \AA$ resolution X-ray structure of the $\mathrm{Vpr} / \mathrm{UBA} 2$ domain complex. Interestingly, our structural findings reveal that hHR23A interacts with the DCAF1-binding and not the substrate-binding Vpr surface, unlike other previously characterized $\mathrm{Vpr}$ targets involved in post-replication DNA repair pathways, and that this interaction antagonizes DCAF1 binding. We also show that Vpr binds hHR23A in a complex with XPC. Thus, our findings establish that HIV-1 Vpr interfaces with DNA repair pathways not only via CRL4 ${ }^{\text {DCAF1 }}$ E3 and proteasomal degradation of targets, but also through the hHR23/XPC complex, suggesting the possibility of an alternative to the ubiquitin ligase route. Our findings further illustrate how HIV-1 Vpr acts as a versatile structural adapter that targets diverse DNA repair pathways.

\section{Results}

Vpr/hHR23A complex preparation and NMR and X-ray studies. Previous work from our laboratory demonstrated that the C-terminal half of hHR23A, hHR23 $\mathrm{A}_{223-363}$, comprising both $\mathrm{XPCB}$ and UBA2 domains, directly binds $\mathrm{Vpr}^{37}$. Although the $\mathrm{Vpr}_{1-79} / \mathrm{hHR} 23 \mathrm{~A}_{223-363}$ complex could be prepared after coexpression, purification in amounts for structural studies proved challenging, and screening by ${ }^{1} \mathrm{H}-{ }^{15} \mathrm{~N}$ HSQC NMR spectroscopy revealed a mixture of complex and free hHR23 $\mathrm{A}_{223-363}$ proteins ${ }^{37}$. Therefore, in order to prepare a homogeneous sample of the complex, we generated $\mathrm{Vpr}_{1-79}$ and hHR23 $\mathrm{A}_{223-363}$ fusion constructs, connecting $\mathrm{Vpr}$ to either the $\mathrm{N}$ - or C-terminus of the double domain construct of hHR23A by a six amino acid (GGS) linker (L) (details are provided in the "Methods" section). Assessment of the structural properties of the linked proteins was performed by ${ }^{1} \mathrm{H}-{ }^{15} \mathrm{~N}$ HSQC spectroscopy. While the spectrum of the C-terminal fusion construct, hHR23 $\mathrm{A}_{223-363}-\mathrm{L}-\mathrm{Vpr}_{1-79}$, exhibited severe NMR line-broadening and loss of ${ }^{1} \mathrm{H}-{ }^{15} \mathrm{~N}$ HSQC cross peaks (Supplementary Fig. 1a) compared to the free hHR23a protein, the spectrum of the N-terminal fusion construct, $\mathrm{Vpr}_{1-79}$-L-hHR23A $223-363$ (Supplementary Fig. 1b), was of sufficient quality to proceed with resonance assignments and structure determination. This suggested that the six amino acid containing linker at the end of $\mathrm{Vpr}_{1-79}$ was long enough to allow for correct positioning of the two proteins in the complex, while it was most likely too short to permit correct complex formation when used to connect the C-terminus of hHR23A to the $\mathrm{N}$-terminus of Vpr. Good agreement for the amide resonances in the ${ }^{1} \mathrm{H}-{ }^{15} \mathrm{~N}$ HSQC spectrum of the $\mathrm{Vpr}_{1-79}-\mathrm{L}-\mathrm{hHR} 23 \mathrm{~A}_{223-363}$ linked and the $\mathrm{Vpr}_{1-79}$ and $\mathrm{hHR} 23 \mathrm{~A}_{223-363}$ co-expressed complexes are noted (Supplementary Fig. 1c), confirming that essentially identical complexes are formed by the linked and unlinked proteins.

NMR assignments for the linked $\mathrm{Vpr}_{1-79}-\mathrm{L}-\mathrm{hHR} 23 \mathrm{~A}_{223-363}$ construct, in which $\mathrm{Vpr}$ is intramolecularly bound to hHR23A $223-363$, were obtained for $97 \%$ of the backbone resonances and $92 \%$ of all side chain resonances. Chemical shift-derived secondary structure elements for Vpr and the XPCB and UBA2 domains (Supplementary Fig. 1d) are essentially the same as those for free $\mathrm{Vpr}^{36}$ and the individual hHR23A domains ${ }^{38-40}$. The amide resonances for the $13 \mathrm{~N}$-terminal $\mathrm{Vpr}$ residues, those of the (GGS) $)_{2}$ linker, the first seven residues (223-229) before XPCB, the flexible linker residues (288-314) between XPCB and UBA2 and the last residues (360-363) following UBA2 are sharp and exhibit only small secondary shifts, compared to random coil values (Supplementary Fig. 1d), indicating that these regions are mobile and essentially adopt random coil conformations. Comparison between assignments for the Vpr-bound portion of hHR23A in $\mathrm{Vpr}_{1-79}-\mathrm{L}$ hHR23 $A_{223-363}$ and free hHR23 $A_{223-363}$ permitted unequivocal delineation of the Vpr binding site on hHR23A by chemical shift mapping. This refined and completed the details of our earlier binding site determination ${ }^{37}$, which for the non-linked Vpr- 
a
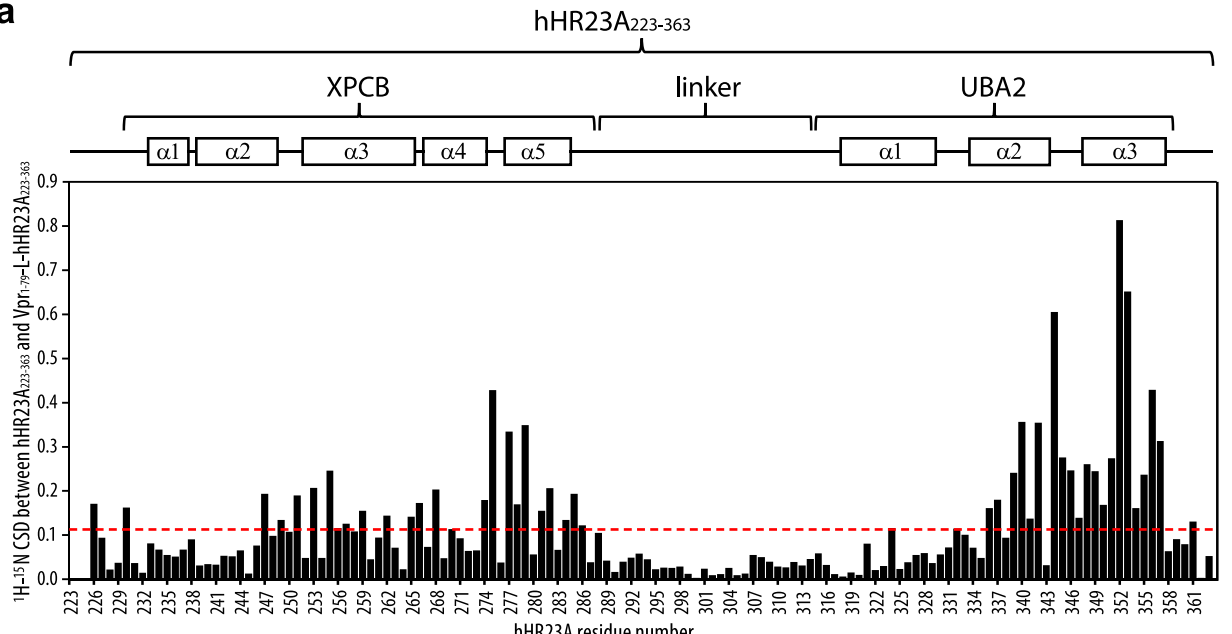

hHR23A residue number

b

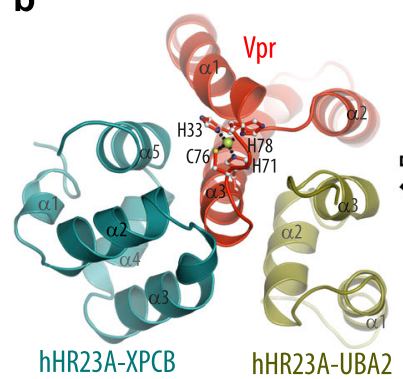

C

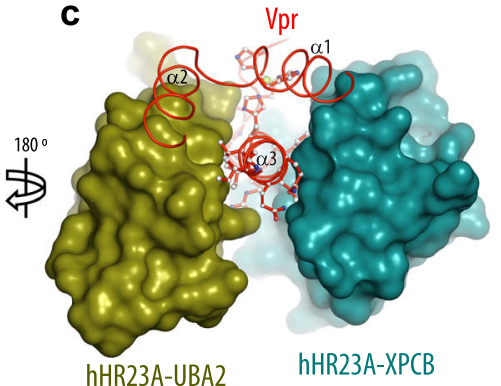

d

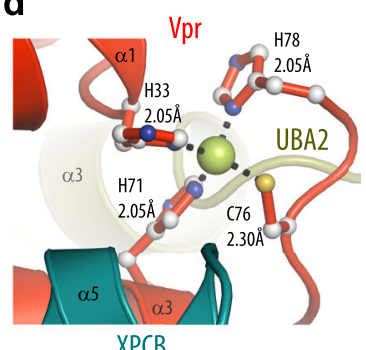

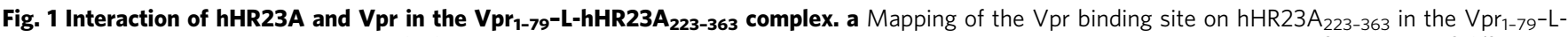
hHR23A $223-363$ complex by NMR. The ${ }^{1} \mathrm{H}^{15}{ }^{15} \mathrm{~N}$-combined chemical shift differences (CSDs), calculated using the equation, $\left(\Delta \delta_{\mathrm{HN}}{ }^{2}+\left(\Delta \delta_{\mathrm{N}} / 6\right)^{2}\right)^{1 / 2}($ for all amino acids, but prolines) or $\left|\Delta \delta_{\mathrm{H \alpha}}\right|$ (for prolines), with $\Delta \delta_{\mathrm{HN}}, \Delta \delta_{\mathrm{N}}$ and $\Delta \delta_{\mathrm{H \alpha}}$ representing the ${ }^{1} \mathrm{HN},{ }^{15} \mathrm{~N}$ and $\mathrm{H}_{\alpha}$ chemical shift differences between resonance frequencies of free hHR23A $223-363$ and those in the $\mathrm{Vpr}_{1-79}-\mathrm{L}-\mathrm{hHR} 23 \mathrm{~A}_{223-363}$ complex, are plotted along the linear amino acid sequence. The average CSD value is drawn in a red dotted line. Domain and secondary structure elements are schematically indicated at the top. $\mathbf{b}, \mathbf{c}$ Lowest energy NMR conformer of the $\mathrm{Vpr}_{1-79}-\mathrm{L}-\mathrm{hHR} 23 \mathrm{~A}_{223-363}$ complex in ribbon representation (b) and worm $\left(\mathrm{Vpr}_{1-79}\right)$ and space filling representation (hHR23A $\mathrm{A}_{223-363}$ ) (c). d Close-up view of the $\mathrm{Zn}^{2+}$-binding motif. Distances between the $\mathrm{Zn}^{2+}$ ion and His and Cys atoms are labeled. Helices are labeled as $\alpha 1, \alpha 2$ and $\alpha 3$.

complexed hHR23A had been largely based on comparing amide backbone assignments. We observed significant chemical shift differences (CSDs) (>0.2 ppm) for resonances associated with all helices, but $a 1$, in the XPCB domain, confirming that most of $\mathrm{XPCB}$ is involved in binding. Even larger CSDs (>0.3 ppm) were observed for amide resonances belonging to the UBA2 domain, in particular for resonances associated with helices $\alpha 2$ and $\alpha 3$. Resonances of amino acids in the first helices of both, the XPCB and UBA2 domains, exhibited only very small CSDs, indicating that they are marginally affected by the interaction with $\mathrm{Vpr}$. The magnitude and organization of all CSDs along the hHR23 $\mathrm{A}_{223-363}$ amino acid sequence are summarized in Fig. 1a.

Crystallization of $\mathrm{Vpr}_{1-79}-\mathrm{L}-\mathrm{hHR} 23 \mathrm{~A}_{223-363}$ yielded crystals belonging to the $\mathrm{P} 3{ }_{1} 21$ space group. Surprisingly, no electron density could be located for the XPCB domain, most likely resulting from its loss due to proteolytic cleavage during crystallization. Mass spectrometry of the crystals confirmed the presence of the UBA2 domain but provided no evidence for the presence of the XPCB domain (Supplementary Fig. 2). Crystals contained four $\mathrm{Vpr}$ and two hHR23A-UBA2 molecules in the asymmetric unit, and the structure was solved using the phases from the native $\mathrm{Vpr} \mathrm{Zn}^{2+}$ anomalous signal, collected at the $\mathrm{Zn}^{2+}$ edge $(9.65 \mathrm{keV})$ to $3.0 \AA$ resolution and further extended to $2.2 \AA$ from a dataset collected at the Se edge $(12.65 \mathrm{keV})$. The model was refined using the program BUSTER ${ }^{41,42}$, followed by manual model building in $\operatorname{Coot}^{43}$, to a final $R_{\text {work }} / R_{\text {free }}$ value of $19.7 /$ $21.6 \%$, respectively (see Table 1 ).
Overall structure of the $\mathrm{Vpr} / \mathrm{hHR} 23 \mathrm{~A}$ complex. The NMR solution structure of the linked $\mathrm{Vpr}_{1-79}-\mathrm{L}-\mathrm{hHR} 23 \mathrm{~A}_{223-363} \mathrm{com}$ plex (Fig. 1b-d) was calculated on the basis of 7650 NOE-derived distance constraints, including $81 \mathrm{Vpr} / \mathrm{XPCB}$ and $257 \mathrm{Vpr} / \mathrm{UBA} 2$ intermolecular distance constraints, and 330 dihedral angle constraints. The final 55-conformer ensemble of the complex satisfies all experimental constraints and displays excellent covalent geometry with atomic root-mean-square differences (r.m.s.d.) of $0.67 \pm 0.06$ and $1.13 \pm 0.05 \AA$ for the backbone and all heavy atoms, respectively, relative to their mean position (Table 2 and Supplementary Fig. 1e). The Vpr structure consists of three antiparallel $a$-helices packed into a helical bundle, and very few conformational changes are noted in the hHR23A-bound Vpr, compared to the free structure ${ }^{36}$. Vpr's helix $\alpha 3$ is buried between the XPCB and UBA2 domains (Fig. 1b, c). Further interactions in the complex involve Vpr's $\alpha 2$ and UBA2's a 3 helices. In the X-ray structure of the Vpr/UBA2 domain complex, the features of the interaction interface are very similar to those observed in the NMR structure: the backbone r.m.s.d. between the NMR and $\mathrm{X}$-ray structures for residues 14-78 of Vpr and residues 315-359 of UBA2 is $1.07 \AA$ (Supplementary Fig. 1f).

The X-ray structure of Vpr in complex with the UBA2 domain revealed atomic details of Vpr's $\mathrm{Zn}^{2+}$-binding motif, which had not been resolved previously in either the X-ray structure of the DDB1/DCAF1/Vpr/UNG2 complex ${ }^{23}$ (PDB: 5JK7) or in the NMR structure of free $\mathrm{Vpr}^{36}$. The $\mathrm{Zn}^{2+}$ atom is coordinated by residues His33, His71, Cys76 and His78 at the C-terminal end of 
Table 1 Data collection and refinement statistics.

\begin{tabular}{|c|c|c|}
\hline & $\begin{array}{l}\mathrm{Vpr}_{1-79} / \mathrm{hHR} 23 \mathrm{~A}- \\
\text { UBA2 (Native)a }\end{array}$ & $\begin{array}{l}\mathrm{Vpr}_{1-79} / \mathrm{hHR} 3 \mathrm{R}- \\
\text { UBA2 (Zinc) }\end{array}$ \\
\hline \multicolumn{3}{|l|}{ Data collection } \\
\hline Space group & $\mathrm{P}_{1} 21$ & $\mathrm{P}_{1} 21$ \\
\hline \multicolumn{3}{|l|}{ Cell dimensions } \\
\hline$a, b, c(\AA)$ & $130.15,130.15,81.31$ & $\begin{array}{l}129.48 \\
129.48,80.77\end{array}$ \\
\hline$\alpha, \beta, \gamma\left(^{\circ}\right)$ & $\begin{array}{l}90.00, \\
90.00,120.00\end{array}$ & $\begin{array}{l}90.00, \\
90.00,120.00\end{array}$ \\
\hline Resolution $(\AA)$ & $50-2.29$ & $50-3.10$ \\
\hline$R_{\text {merge }}$ & $8.6(247.2)$ & $19.5(387.1)$ \\
\hline$R_{\mathrm{sym}}$ & $7.7(214.4)$ & $18.9(387.1)$ \\
\hline $\mathrm{I} / \sigma(\mathrm{I})$ & $9.1(0.7)$ & $12.8(0.7)$ \\
\hline $\mathrm{CC}_{1 / 2}$ & $99.9(23.0)$ & $99.9(22.6)$ \\
\hline Completeness (\%) & $90.6(86.3)$ & $99.9(98.7)$ \\
\hline Redundancy & $3.5(2.7)$ & $18.0(13.6)$ \\
\hline \multicolumn{3}{|l|}{ Refinement } \\
\hline $\mathrm{FOM}(\mathrm{SAD})^{\mathrm{C}}$ & & 0.37 \\
\hline Resolution $(\AA)$ & $40-2.29$ & $40-3.0$ \\
\hline No. of reflections & 29,904 & 15,409 \\
\hline$R_{\text {work }} / R_{\text {free }}$ & $19.7 / 21.6$ & $21.3 / 23.7$ \\
\hline No. of atoms of protein & 2841 & 2841 \\
\hline$B$ factors $\left(\AA^{2}\right)$ & 34 & 50 \\
\hline \multicolumn{3}{|l|}{ R.M.S. deviations } \\
\hline Bond lengths $(\AA)$ & 0.05 & 0.08 \\
\hline Bond angles $\left({ }^{\circ}\right)$ & 1.1 & 0.93 \\
\hline $\begin{array}{l}\text { Numbers in parentheses correspo } \\
\text { respectively). } \\
\text { aData set was recorded at a wav } \\
\text { bData set was recorded at a wav } \\
\text { cPhasing was performed using th }\end{array}$ & $\begin{array}{l}\text { id to the highest resolution sh } \\
\text { length of } 0.97946 \AA \text {. } \\
\text { length of } 1.27368 \AA \text {. } \\
\text { program Crank (CCP4i suite) }\end{array}$ & I (2.43-2.29 and 3.18-3.10 $\AA$ \\
\hline
\end{tabular}

Vpr's helix a3 (Fig. 1d and Supplementary Fig. 1f). His78 is the penultimate amino acid in the current $\mathrm{Vpr}$ construct and the $\mathrm{Zn}$ binding cluster is important for maintaining the global and local architecture of Vpr.

Vpr/UBA2 domain binding interface. The binding interfaces on both Vpr and the UBA2 domain are extensive, burying a surface area of $760 \AA^{2}$. Amino acids F34, W38, L42, I46, I63, L67 and I70 (helices $\alpha 2$ and $\alpha 3$ ) of Vpr pack against UBA2 residues F354, L350, A343, A340, Q339 and L336 (helices $\alpha 3$ and $\alpha 2$ ) in an antiparallel manner (Fig. 2a-c and Supplementary Fig. 3a-c). In addition, residues at the $\mathrm{C}$ - and N-terminal ends of Vpr's $a 2$ and $\alpha 3$ helices, H45, Y50, R73 and H71 and UBA2's $\alpha 2$ and $\alpha 3$ helices, Q339, E345, N347 and N359, engage in hydrogen bonding and ionic interactions that contribute to binding (Fig. 2a). Amino acids in the Vpr-UBA2 intermolecular interface are listed in Supplementary Table 1 . Within the UBA2 domain, an intramolecular NH..pi H-bond between the Q358 $\mathrm{N}_{\varepsilon 2} \mathrm{H}_{2}$ amino group and the F354 aromatic ring is seen in the structure (Fig. 2a, b, orange dashed line), evidenced by the presence of intramolecular NOEs (Supplementary Fig. 3e) and a very large ring currentinduced upfield shift of the Q358 amino group hydrogens (6.30, $6.46 \mathrm{ppm}$ ). The F354 aromatic ring is inserted into a small hydrophobic pocket lined by the side chains of Vpr's F34, W38 and L67 (Fig. 2a-c). These binding interfaces in the $\mathrm{Vpr}_{1-79}-\mathrm{L}-$ hHR23A $\mathrm{A}_{223-363}$ complex are clearly delineated by intermolecular NOEs (Fig. 2d, e, right and Supplementary Fig. 3e, f). The 3D NOESY data of the $\mathrm{Vpr}_{1-79}-\mathrm{L}-\mathrm{hHR} 23 \mathrm{~A}_{223-363}$ complex were cross-checked and confirmed by comparison with the 3D NOESY data recorded on a sample of free hHR23 $\mathrm{A}_{223-363}$ (Fig. 2e, left). As illustrated, intermolecular NOEs between the A340 $\mathrm{H}_{\mathrm{N}}$ of hHR23A and L67 $\mathrm{H}_{\delta}$ of $\mathrm{Vpr}$ are only present in the spectrum of the $\mathrm{Vpr}_{1-79}-\mathrm{L}$-hHR23A $\mathrm{A}_{223-363}$ complex (Fig. 2e, right) and not in
Table 2 Statistics for the final $\mathbf{5 5}$ conformer ensemble of the Vpr $_{\text {1-79-L-hHR23A }}$ 223-363 complex.

\begin{tabular}{|c|c|}
\hline \multicolumn{2}{|l|}{ Number of NOE distance constraints } \\
\hline Intra-residue $(i-j=0)$ & 2377 \\
\hline Sequential $(i-j=1)$ & 1806 \\
\hline Medium range $(2 \leq i-j \leq 4)$ & 1972 \\
\hline Vpr long range $(i-j \geq 5)$ & 327 \\
\hline hHR23A long range $(i-j \geq 5)$ & 830 \\
\hline Vpr-XPCB intermolecular & 81 \\
\hline Vpr-UBA2 intermolecular & 257 \\
\hline Total & 7650 \\
\hline \multicolumn{2}{|l|}{ Number of dihedral angle constraints } \\
\hline$\phi$ & 166 \\
\hline$\psi$ & 164 \\
\hline Total & 330 \\
\hline \multicolumn{2}{|l|}{ Structural quality } \\
\hline \multicolumn{2}{|l|}{ Violations $^{\mathrm{a}}$} \\
\hline Distance constraints $(\AA)$ & $0.009 \pm 0.001$ \\
\hline Dihedral angle constraints (deg) & $0.276 \pm 0.056$ \\
\hline \multicolumn{2}{|c|}{ Deviation from idealized covalent geometry } \\
\hline Bond lengths $(\AA)$ & $0.001 \pm 0.000$ \\
\hline Bond angles (deg) & $0.385 \pm 0.003$ \\
\hline Improper torsions (deg) & $0.216 \pm 0.006$ \\
\hline \multicolumn{2}{|c|}{$\begin{array}{l}\text { Average root-mean-square deviation of atomic } \\
\text { coordinates }(\AA)^{b, c}\end{array}$} \\
\hline Backbone heavy atoms & $0.67 \pm 0.06$ \\
\hline All heavy atoms & $1.13 \pm 0.05$ \\
\hline \multicolumn{2}{|l|}{ Ramachandran analysis (\%) ${ }^{\mathrm{c}}$} \\
\hline Most favorable regions & $88.8 \pm 1.8$ \\
\hline Additional allowed regions & $10.5 \pm 1.9$ \\
\hline Generously allowed regions & $0.7 \pm 0.5$ \\
\hline Disallowed regions & $0 \pm 0.1$ \\
\hline
\end{tabular}

that of the free hHR23 $\mathrm{A}_{223-363}$ (shown by two $\mathrm{X}$ symbols in Fig. 2e, left).

The Vpr/UBA2 interface was validated by mutagenesis of the involved amino acids on both $\mathrm{hHR} 23 \mathrm{~A}_{\mathrm{FL}}$ and $\mathrm{Vpr}_{\mathrm{FL}}$. Several mutants of hHR23 $\mathrm{A}_{\mathrm{FL}}$ were examined: the E345A/N347A mutant was tested, since E345 and N347 form a salt bridge and a hydrogen bond with Vpr Y50 and H45, respectively (Supplementary Fig. 3a); the F354A/Q358A mutant was assessed since the aromatic side chain is integral to the UBA2 structure and thus it was expected that this mutation would compromise the integrity of the fold (Fig. 2a-c and Supplementary Fig. 3b); the single Q339A mutant was tested, since Q339 forms a salt bridge with $\mathrm{R} 73$ of $\mathrm{Vpr}$ at the periphery of the $\mathrm{Vpr}-\mathrm{UBA} 2$ interface (Fig. 2a-c and Supplementary Fig. 3c). The two double mutants, E345A/N347A and F354A/Q358A, abolished Vpr binding (Fig. 2f, top panel), while the single mutant Q339A had little effect. On the Vpr side, H45A, Y50S, R73A and the double mutant $\mathrm{L} 67 \mathrm{~A} / \mathrm{I} 70 \mathrm{~A}$ were tested. All of these $\mathrm{Vpr}_{\mathrm{FL}}$ mutants exhibited impaired binding to hHR23 $\mathrm{A}_{\mathrm{FL}}$ (Fig. 2f, bottom panel). Thus, all these residues are implicated in structural integrity or binding and changing them either destabilizes Vpr's fold or removes important contacts, thereby indirectly or directly interfering with binding.

Previous work in our ${ }^{37}$ and other ${ }^{25}$ laboratories showed that hHR23A's UBA2 domain, but not the UBA1 domain, is involved in Vpr binding. Indeed, the structure presented here and our sitedirected mutagenesis experiments validate this model. Most of the UBA2 residues that are important for the interaction with $\mathrm{Vpr}$ 
a

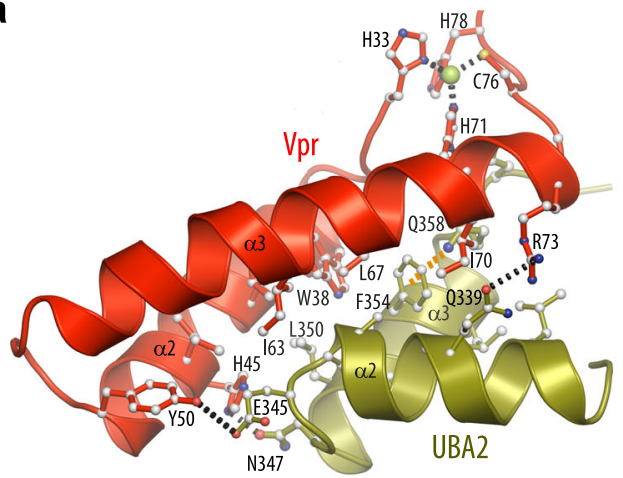

b
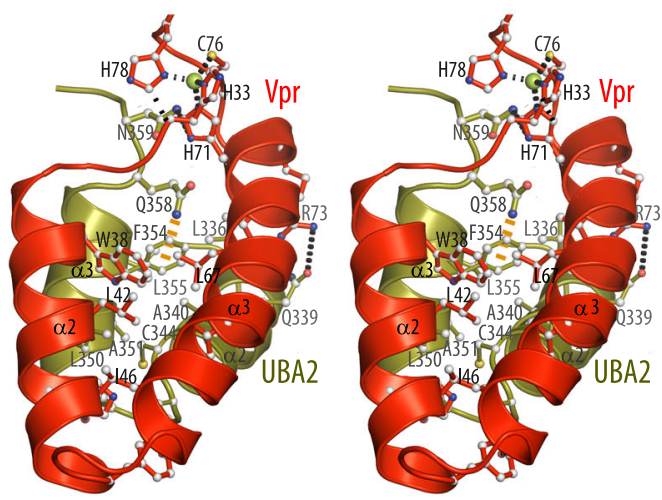

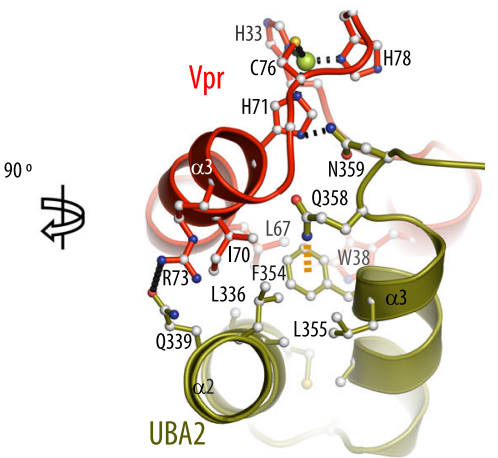

C

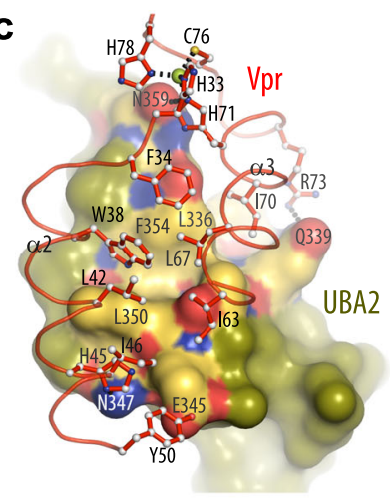

d

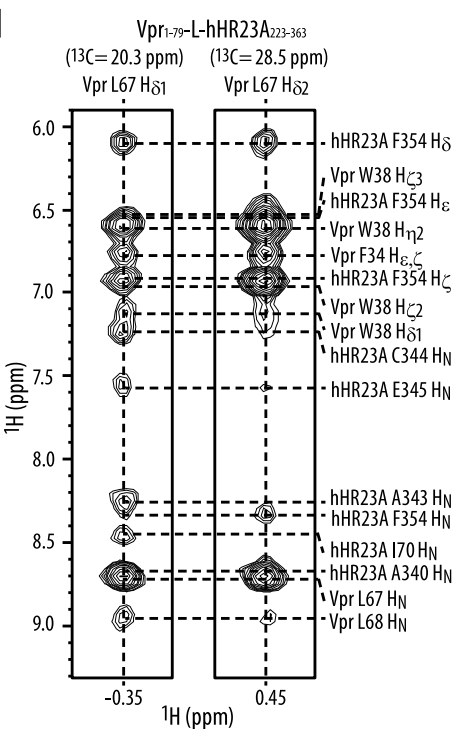

e

$$
\begin{gathered}
\text { hHR23A } 223-363 \\
(15 \mathrm{~N}=121.2 \mathrm{ppm})
\end{gathered}
$$
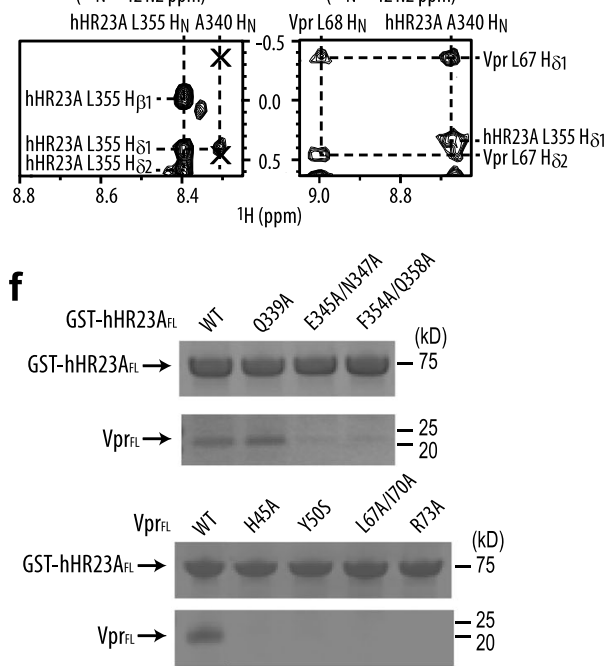

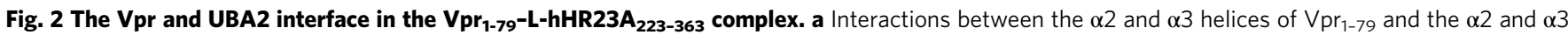
helices in the UBA2 domain in two orientations, $\mathbf{b}$ stereoview with $\mathrm{Vpr}_{1-79}$ at the front. The $\mathrm{Vpr} \alpha 1$ and UBA2 $\alpha 1$ helices that are not involved in the $\mathrm{Vpr} /$ UBA2 interface are omitted for clarity. In $\mathbf{a}$ and $\mathbf{b} \mathrm{Vpr}$ and UBA2 are shown in red and olive ribbon representation, respectively, with selected sidechains in ball-and-stick representation. c worm (Vpr) and surface (UBA2) representations, with the $\mathrm{Vpr} \alpha 1$ helix and residues 51-62 omitted for clarity. Vpr is shown in red and UBA2 in olive. Selected $\mathrm{H}$-bonds and the $\mathrm{Zn}$-coordinating bonds are indicated by dotted black lines and the $\mathrm{NH}_{2}$-pi $\mathrm{H}_{\text {-bond in orange. Interacting }}$ residues in UBA2 are labeled and colored in gold (hydrophobic), red (negatively charged) and blue (positively charged). d Selected regions of the 3D

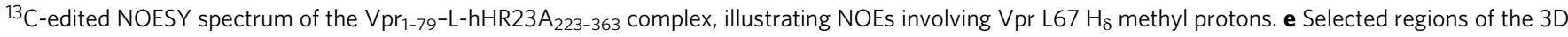

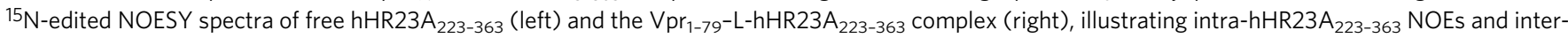
molecular NOEs between hHR23A $223-363$ and $\mathrm{Vpr}_{1-79}$ for the $\mathrm{A} 340 \mathrm{H}_{\mathrm{N}}$ proton. The missing intermolecular cross-peaks in the free hHR23 $\mathrm{A}_{223-363} \mathrm{Spectrum}$ are indicated by the symbols X. f GST-pull down data for WT and mutant hHR23A FL proteins (top) and Vpr FL (bottom). Source data are provided as a Source Data file. Three independent experiments were performed yielding similar results. 
a
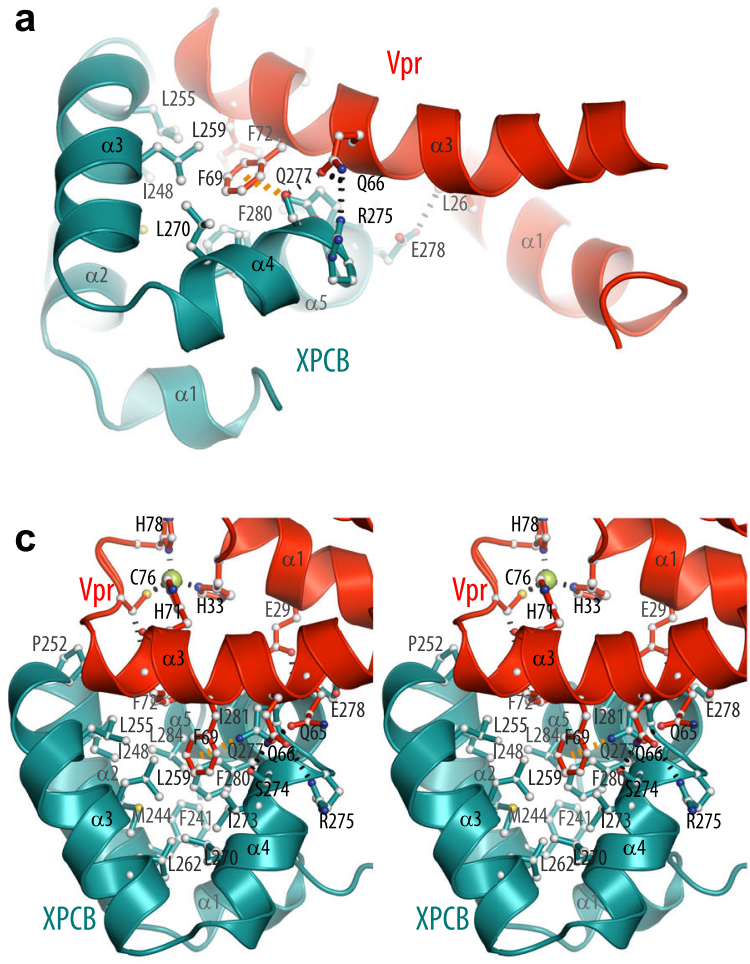

b
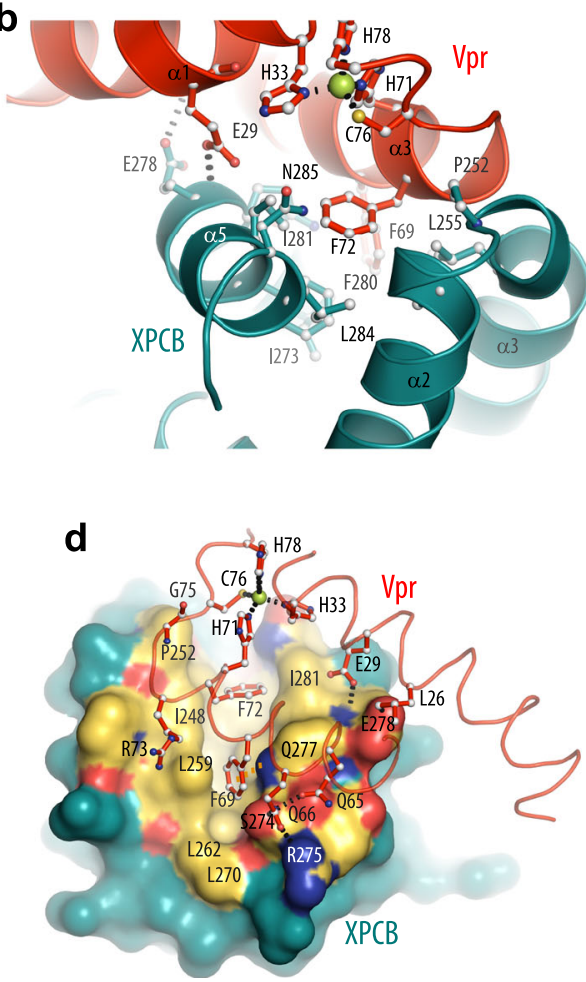

e

$$
\begin{array}{ll}
\text { hHR23A223-363 } & \text { Vpri-79-L-hHR23A223-363 } \\
(13 \mathrm{C}=11.7 \mathrm{ppm}) & (13 \mathrm{C}=12.6 \mathrm{ppm}) \\
\text { hHR23A } 281 \mathrm{H}_{\delta 1} & \text { hHR23A } 1281 \mathrm{H} / \mathrm{H} 1
\end{array}
$$

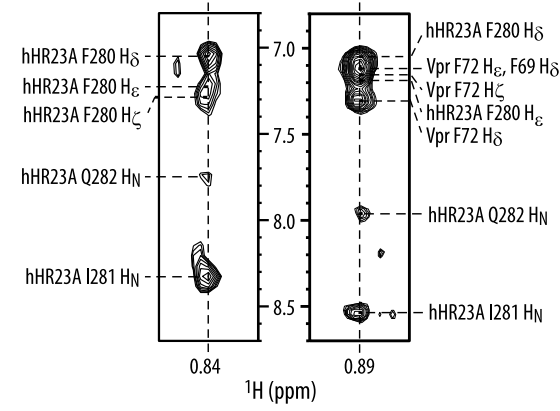

$\mathbf{f}$

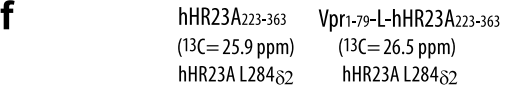
hHR23AL $284 \delta 2 \quad$ hHR23A L284 2

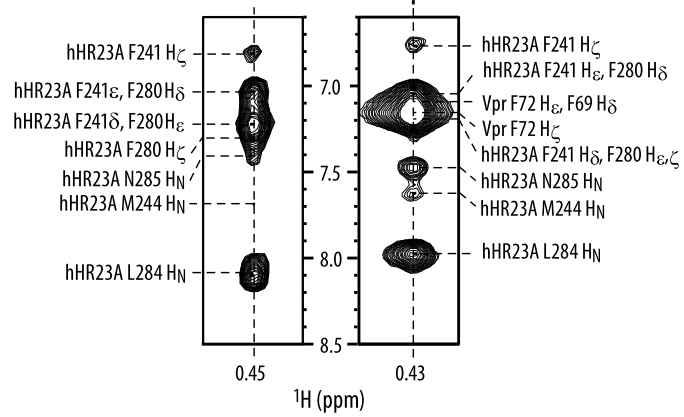

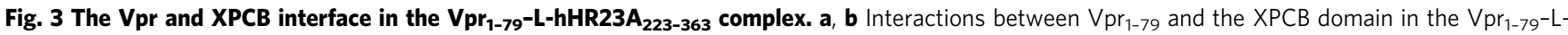
hHR23A $\mathrm{A}_{223-363}$ complex, highlighting residues F69 and F72 in Vpr. c stereoview in a different orientation. In a-c $\mathrm{Vpr}$ and XPCB are shown in red and teal ribbon representation, respectively, with selected sidechains in ball-and-stick representation. $\mathbf{d}$ worm (Vpr) and surface (XPCB) representations. $V p r$ is shown in red and the XPCB domain in teal. Selected amino acids are shown in ball-and-stick representation (Vpr), and $\mathrm{H}$-bonds and the Zn-coordinating bonds are indicated by dotted black lines and the $\mathrm{NH}_{2}$-pi $\mathrm{H}$-bond in orange. Interacting residues in the XPCB domain are labeled and colored in gold (hydrophobic), red (negatively charged) and blue (positively charged). e, $\mathbf{f}$ Selected regions of the 3D ${ }^{13} \mathrm{C}$-edited NOESY spectra of free hHR23A $223-363$ (left) and the $\mathrm{Vpr}_{1-79}-\mathrm{L}-\mathrm{hHR} 23 \mathrm{~A}_{223-363}$ complex (right), illustrating intra-hHR23A $223-363$ NOEs and inter-molecular NOEs between hHR23A $223-363$ and $\mathrm{Vpr}_{1-79}$ for $\mathrm{I} 281 \mathrm{H}_{\delta}(\mathbf{e})$ and $\mathrm{L} 284 \mathrm{H}_{\delta}(\mathbf{f})$ methyl protons.

(Fig. 2a-c) are not present in the UBA1 domain, such as L336 (R179 in UBA1), L350 (R193 in UBA1), F354 (Y197 in UBA1), Q339 (A182 in UBA1), E345 (Y188 in UBA1), Q358 (G201 in UBA1) and N359 (I202 in UBA1) (Supplementary Fig. 3d).

Vpr/XPCB domain binding interface. The XPCB domain interacts with the $\alpha 1$ and $\alpha 3$ face of $\mathrm{Vpr}$ (Fig. 1b, c), burying a surface area of $670 \AA^{2}$, somewhat smaller than the UBA2-Vpr interface. The binding interface between $\mathrm{Vpr}$ and the XPCB domain (Fig. 3a-d) in the NMR structure of $\mathrm{Vpr}_{1-79}-\mathrm{L}$ hHR23 $A_{223-363}$ was identified by several intermolecular NOEs in the 3D NOESY spectrum (Fig. 3e, f, right and Supplementary Fig. 3g), cross-checked against NOEs present in the 3D NOESY data of free hHR23A $223-363$ (Fig. $3 \mathrm{e}, \mathrm{f}$, left) where the intermolecular NOEs between the hHR23A I281 ${ }_{\mathrm{H} \delta 1} / \mathrm{L}_{28} 4_{\mathrm{H} \delta 2}$ and the Vpr F72/F69 aromatic resonances are missing. The main contacts involve the phenyl rings of F69 and F72 of Vpr that insert into a hydrophobic cleft on XPCB (Fig. 3a-d). In the current structure, Vpr F69 is surrounded by a cluster of XPCB hydrophobic residues that include I248, L255, L259, L270, I273, F280, I281 and L284. The Vpr F72 side chain is bordered by XPCB I248, P252, L255, F280, I281 and L284. In addition, an intermolecular NH...pi interaction is present between a side-chain $\mathrm{N}_{\varepsilon 2} \mathrm{H}_{2}$ proton of XPCB's Q277 and the phenyl ring of Vpr's F69 (Fig. 3a, c, orange dashed line), consistent with the observation of the intermolecular NOEs (Supplementary Fig. 3g) and the large ring 
a

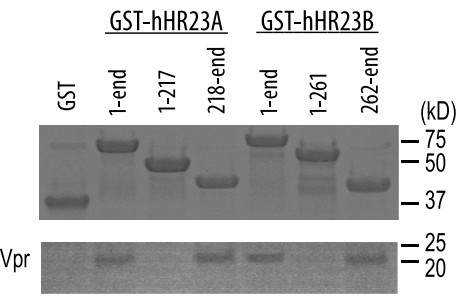

b

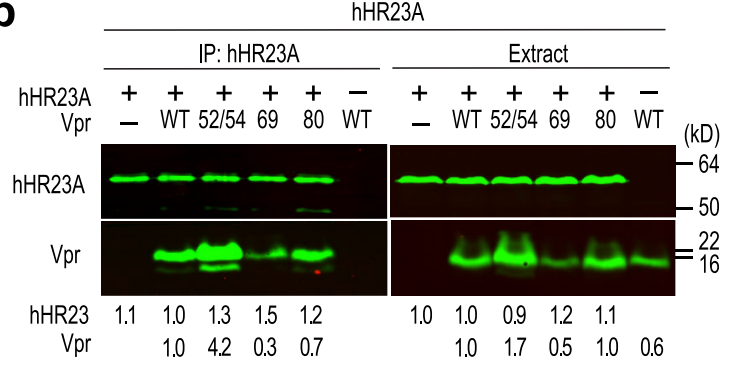

hHR23B

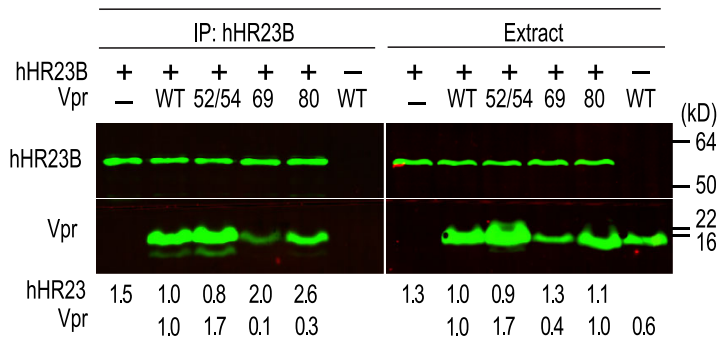

C

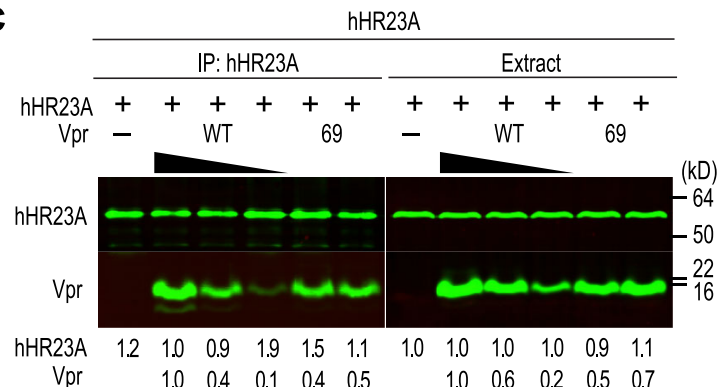

d

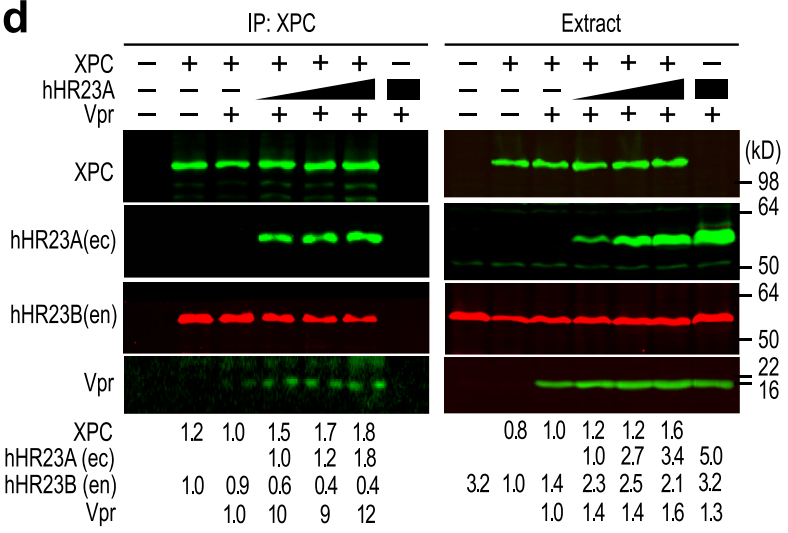

current-induced upfield shift of one of the Q277 $\mathrm{N}_{\varepsilon 2} \mathrm{H}_{2}$ protons from the free positions of 7.36 and $6.55 \mathrm{ppm}$ to 7.01 and $6.19 \mathrm{ppm}$. Several other H-bonds between Vpr and the XРCB domain are also observed: E29-E278, Q66-R275 and Q66-S274 (Fig. 3a-d). All residues directly involved in the Vpr-XPCB intermolecular interface, as evidenced by inter-molecular NOEs, are summarized in Supplementary Table 2. Notably, F69 is a key
Fig. $4 \mathrm{Vpr}$ binds both the hHR23A and hHR23B isoforms and the hHR23/ XPC DNA recognition complex. a Recombinant GST-hHR23A, residues 1-363, 1-217 and 218-363 and GST-hHR23B, residues 1-409, 1-261 and 262-409 were used in GST-pull down experiments with recombinant $\mathrm{Vpr}_{\mathrm{FL}}$. Three replicates were performed for each experiment yielding similar results. b Vpr mutant binding to hHR23A (top) and hHR23B (bottom).

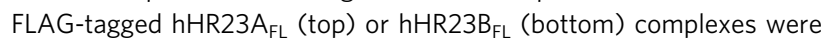
immunoprecipitated, via FLAG-tag, from HEK293T cell extracts transiently co-expressing the respective $\mathrm{hHR} 23$ isoform and $\mathrm{HA}$-tagged $\mathrm{Vpr}_{\mathrm{FL}}$ wild type (WT) or with the following mutations: D52R/W54R (52/54), F69A (69), R80A (80). Cell extracts and hHR23 immune complexes were analyzed by immunoblotting with antibodies specific for the FLAG and HA epitope tags, to detect the ectopically expressed FLAG-hHR23A, FLAGhHR23B and HA-Vpr proteins. c Vpr F69A mutant binding to hHR23A. Expression levels of the HA-Vpr (F69A) and Vpr WT were varied by transfecting different amounts of plasmids expressing these proteins and their binding to FLAG-hHR23A was assessed as described above. $\mathbf{d} \mathrm{Vpr}$ binds the hHR23/XPC complex. FLAG-tagged XPC, HA-tagged hHR23A and myc-tagged $V_{p_{F}}$ were expressed alone or in combinations in HEK293 $T$ cells. Cell extracts and XPC immune complexes were analyzed by immunoblotting as described above except that the endogenously expressed hHR23B(en) protein was detected with an antibody specific for the hHR23B isoform. Immune complexes were revealed with fluorescent secondary antibodies and fluorescent signals quantified using a Li-Cor Odyssey imager. Relative levels of the indicated proteins in cell extracts and immune-complexes are shown. b-d Two biological replicates were performed for each experiment yielding similar results. The biological replicate of the experiment shown in panel $\mathbf{d}$ is shown in Supplementary Fig. 4. a-d Source data are provided as a Source Data file.

Vpr residue for DCAF1 binding ${ }^{23}$, suggesting that DCAF1 and $\mathrm{XPCB}$ binding is mutually exclusive (see also below).

HIV-1 Vpr binds both the hHR23A and hHR23B isoforms. Most studies concerning NER have focused on hHR23B, rather than hHR23A. Given that the XPCB and UBA2 domains of hHR23A and hHR23B exhibit very high amino acid sequence identity (89\%), we hypothesized that Vpr also interacts with hHR23B and investigated this possibility using in vitro and in vivo assays. Indeed, GST pull-down experiments indicated an interaction between $\mathrm{Vpr}$ and hHR23B, with the binding region localized to the C-terminal half of hHR23B (Fig. 4a). In addition, immunoprecipitation experiments revealed the presence of $\mathrm{Vpr}$ in both hHR23A (Fig. 4b, top) and hHR23B (Fig. 4b, bottom) immune complexes isolated from HEK293T cells co-expressing either hHR23 isoform together with Vpr, confirming that Vpr can form a complex with either hHR23 isoform in vivo. We also tested the effect of selected Vpr amino acid changes for binding hHR23A and hHR23B. Significantly, none of the variants tested, including the D52R/W54R one in which key interactions with the DNA repair protein UNG2 were abrogated, or the Vpr R80A variant, which no longer is able to activate G2 DNA damage checkpoint $^{44}$, abolished binding to either hHR23 isoform, consistent with the results of our structural analyses of Vpr-hHR23A complex. In contrast, the Vpr F69A substitution, which disrupts $\mathrm{Vpr}$ binding to $\mathrm{DCAF1}^{23}$, resulted in a reduced Vpr protein steady state level in cell extracts and binding to hHR23 (Fig. 4b). To ascertain whether the latter was simply due to the lower level of Vpr F69A protein in cells, or whether the F69 side chain is indeed critical for hHR23 binding, dose response binding experiments were performed. They were conducted under conditions where Vpr WT and the F69A variant were present at similar levels. Interestingly, similar amounts co-precipitated with hHR23A (Fig. 4c). This suggests that F69 is not critical for hHR23 
binding, and that contacts made by other residues, including Q66 and F72, with the XPCB domain, as well as contacts by W38, L67 and I70 with the UBA2 domain are sufficient to stabilize the complex.

HIV-1 Vpr binds the hHR23A/XPC complex. hHR23 binds $\mathrm{XPC}$, and this complex functions in DNA repair. Independently, hHR23 is also involved in ubiquitin-mediated proteolytic pathways. The latter does not require its association with XPC. To assess whether hHR23 has the potential to connect Vpr to NER, we asked whether Vpr can bind hHR23 in complex with XPC. To this end, XPC was co-expressed with $\mathrm{Vpr}$ in the absence or presence of increasing amounts of hHR23A, and XPC immune complexes were analyzed by immunoblotting for XPC, ectopically expressed hHR23A, endogenously expressed hHR23B isoform, and $\mathrm{Vpr}$.

As shown in Fig. 4d and Supplementary Fig. 4, ectopic hHR23A expression resulted in increased amounts of Vpr coprecipitating with XPC. The low baseline levels of Vpr found in XPC immune complexes in the absence of ectopically expressed hHR23A probably reflect Vpr recruitment into the XPC complex by endogenously expressed $\mathrm{hHR} 23 \mathrm{~A}(\mathrm{~B})$ isoforms, since the endogenous hHR23B is readily detectable in the XPC immune complexes. These findings support the model in which hHR23A(B) connects Vpr to hHR23A(B)/XPC mediated functions but does not exclude its involvement with XPC-independent hHR23 functions.

Vpr interactions with hHR23A and DCAF1 are mutually exclusive. Previous genetic and biochemical studies linked several cellular effects of Vpr to its binding to DCAF1 and, by extension, to the subversion of CRL4 ${ }^{\mathrm{DCAF} 1} \mathrm{E} 3$. Interestingly, comparison between the $\mathrm{Vpr}_{1-79}-\mathrm{L}-\mathrm{hHR} 23 \mathrm{~A}_{223-363}$ (this study) and the UNG2/ $\mathrm{Vpr}_{1-79} / \mathrm{DCAF}_{1045-1396} / \mathrm{DDB} 1$ (PDB: 5JK7) structures reveals that both DCAF1 and hHR23A interact with the $\alpha 3$ helix of $\operatorname{Vpr}$ (Fig. 5l-n and Supplementary Fig. 5a, b). However, engaging these different binding partners results in only a small change in the Vpr structure, namely a slight shift in the Vpr $\alpha 3$ helix (Supplementary Fig. 1g). The buried surface area in the $\mathrm{Vpr}_{1-79} / \mathrm{DCAF}_{1045-1396}$ region is slightly larger than in the $\mathrm{Vpr}_{1-79}$-L-hHR23A $223-363$ complex (1670 vs. $1430 \AA^{2}$, respectively) and removal of DCAF1 residues 1045-1056 permitted purification of DCAF1 without DDB1 $1^{15}$, consistent with previous findings about DDB1 binding ${ }^{23}$. Therefore, our data suggest that Vpr interactions with hHR23A and DCAF1 are competitive, with binding to DCAF1 likely of higher affinity. We hence tested whether DCAF1 $1_{1057-1396}$ can compete and displace hHR23A from $\mathrm{Vpr}_{1-79}$ (Fig. 5a-e). When using $\mathrm{DCAF}_{1057-1396}$ in the presence of a 1:1 (M/M) mixture of $\mathrm{Vpr}_{1-79}$ and $\mathrm{hHR} 23 \mathrm{~A}_{\mathrm{FL}}$ in size exclusion column chromatography, an elution peak for free hHR23A $\mathrm{A}_{\mathrm{FL}}$ (Fig. 5d, peak 4; Fig. 5e, lane 4) and a peak for the $\mathrm{Vpr}_{1-79} / \mathrm{DCAF1}_{1057-1396}$ complex (Fig. 5d, peak 5; Fig. 5e, lane 5) is observed, confirming that $\mathrm{Vpr}$ binds $\mathrm{DCAF} 1_{1057-1396}$ more tightly than hHR23A $\mathrm{AL}_{\mathrm{FL}}$. This again confirms that both these Vprbinding proteins engage the same surface on $\mathrm{Vpr}$ for their interaction, namely helix $\alpha 3$, as structurally confirmed by the best-fit superposition of the $\mathrm{Vpr}_{1-79}-\mathrm{L}-\mathrm{hHR} 23 \mathrm{~A}_{223-363}$ and the $\mathrm{Vpr}_{1-79} /$ DCAF1 $_{1045-1396}$ complex structures (Fig. 5l-n and Supplementary Fig. 5a, b). Both hHR23A $\mathrm{A}_{223-363}$ and DCAF1 $1_{1045-1396}$ utilize hydrophobic residues to contact F69, I70 and F72 and charged residues to form hydrogen bonds with Q65 and Q66 (Fig. 5n). Non-shared interactions include residues from helices $\alpha 2$ and $\alpha 3$ (N41, I74 and R77), which bind the UBA2 domain, and the Vpr N-terminus that wraps around the side of DCAF1's WD40 motif (Fig. 5n). The higher Vpr-binding affinity of DCAF1 was further confirmed by isothermal titration calorimetry (ITC): DCAF1 exhibited a $K_{\mathrm{d}}$ of $116 \pm 47 \mathrm{nM}$ while the hHR23A $K_{\mathrm{d}}$ was $640 \pm 408 \mathrm{nM}$ (Supplementary Fig. 5c, d). The above findings clearly demonstrate that Vpr binding to DCAF1 and hHR23 is mutually exclusive and suggest that hHR23 provides an alternative to the DCAF1 (CRL4 ${ }^{\mathrm{DCAF}}$ E3)-mediated mechanisms for Vpr to exert its function.

The hHR23A $223-363$ interaction with $\mathrm{Vpr}_{1-79}$ spans two of the three surfaces created by Vpr's three helix bundle (Fig. 1b, c), permitting the third surface, particularly Vpr helices $\alpha 1$ and $\alpha 2$, to potentially engage in additional interactions. Previous work by our laboratory demonstrated that UNG2 binds to this third surface (PDB: 5JK7) 23 . We, therefore, tested whether the binary Vpr/hHR23A complex can interact with UNG2. Biochemical experiments, including size exclusion column chromatography, revealed that a ternary UNG2/Vpr/hHR23A complex can indeed be formed (Fig. 5f-k). The UNG2 association with hHR23 is strictly Vpr dependent, since, in the absence of Vpr, the mixture of hHR23A and UNG2 elutes as two individual peaks (Fig. 5i, k). A structural model for such a complex is shown in Fig. 51. Whether the UNG2/Vpr/hHR23A complex forms in HIV-1infected cells and plays a biological role remains to be determined.

\section{Discussion}

HIV-1 Vpr, a small adaptor protein, is the prototype of a large family of primate lentiviral accessory virulence factors. It has been extensively studied over recent years, yet its mode of interaction with target proteins remains poorly understood. The structure of the HIV-1 Vpr/hHR23A complex reported here sheds new light on the molecular mechanisms that underlie the remarkable versatility of this adapter protein and its ability to engage in specific interactions with a large number of structurally unrelated protein partners. Our findings also provide new insights into the cellular mechanisms that $\mathrm{Vpr}$ is capable of targeting.

The core three helix bundle structure of Vpr provides different sides/interfaces to engage interacting proteins, while the unstructured $\mathrm{N}$ - and C-terminal regions potentially provide additional interaction motifs. Previous studies of HIV-1 Vpr complexes with DCAF1 and post-replication DNA repair proteins targeted by $\mathrm{Vpr}$ revealed two distinct $\mathrm{Vpr}$ interfaces: a DCAF1-binding interface and a substrate recruitment interface 20,23 - the latter interacting with multiple targets including UNG2, MUS81 and HLTF ${ }^{17,20,23}$. Strikingly, the current structure of the $\mathrm{Vpr} / \mathrm{hHR} 23 \mathrm{~A}$ complex reveals that hHR23 binds a surface on Vpr that largely overlaps with that engaged by DCAF1. This is remarkable, since hHR23 $\mathrm{A}_{223-363}$ and DCAF $1_{1045-1396}$ exhibit entirely different three-dimensional structures (Fig. 5m and Supplementary Fig. 5a, b). The ability of $\mathrm{Vpr}$ to use the same surface to interact with two structurally unrelated partners, such as hHR23A and DCAF1, is unusual, possibly indicating that other proteins may potentially be engaged.

Both hHR23A and DCAF1 bind the solvent exposed sides of Vpr's $a 3$ helix, with only a few contacts that are specific to either UBA2 of hHR23A, such as N41 located in the a2 helix and I74 and R77 in the $\alpha 3$ helix, or to DCAF1, such as Vpr's N-terminal region (Fig. 5n). These divergent, but important contacts probably contribute to the slightly higher affinity of $\mathrm{DCAF}_{1045-1396}$ for $\mathrm{Vpr}$, compared to that of hHR23A $\mathrm{AL}_{\mathrm{FL}}$. A similar strategy is evident in Vpr's recruitment of substrates: Vpr utilizes both common and distinctive interfaces to recruit different postreplication DNA repair proteins to the CRL4 ${ }^{\mathrm{DCAF} 1} \mathrm{E} 3$ ligase for ubiquitin-dependent proteasomal degradation ${ }^{17,20,23}$. This structural adaptation may also be important in the specific recruitment 

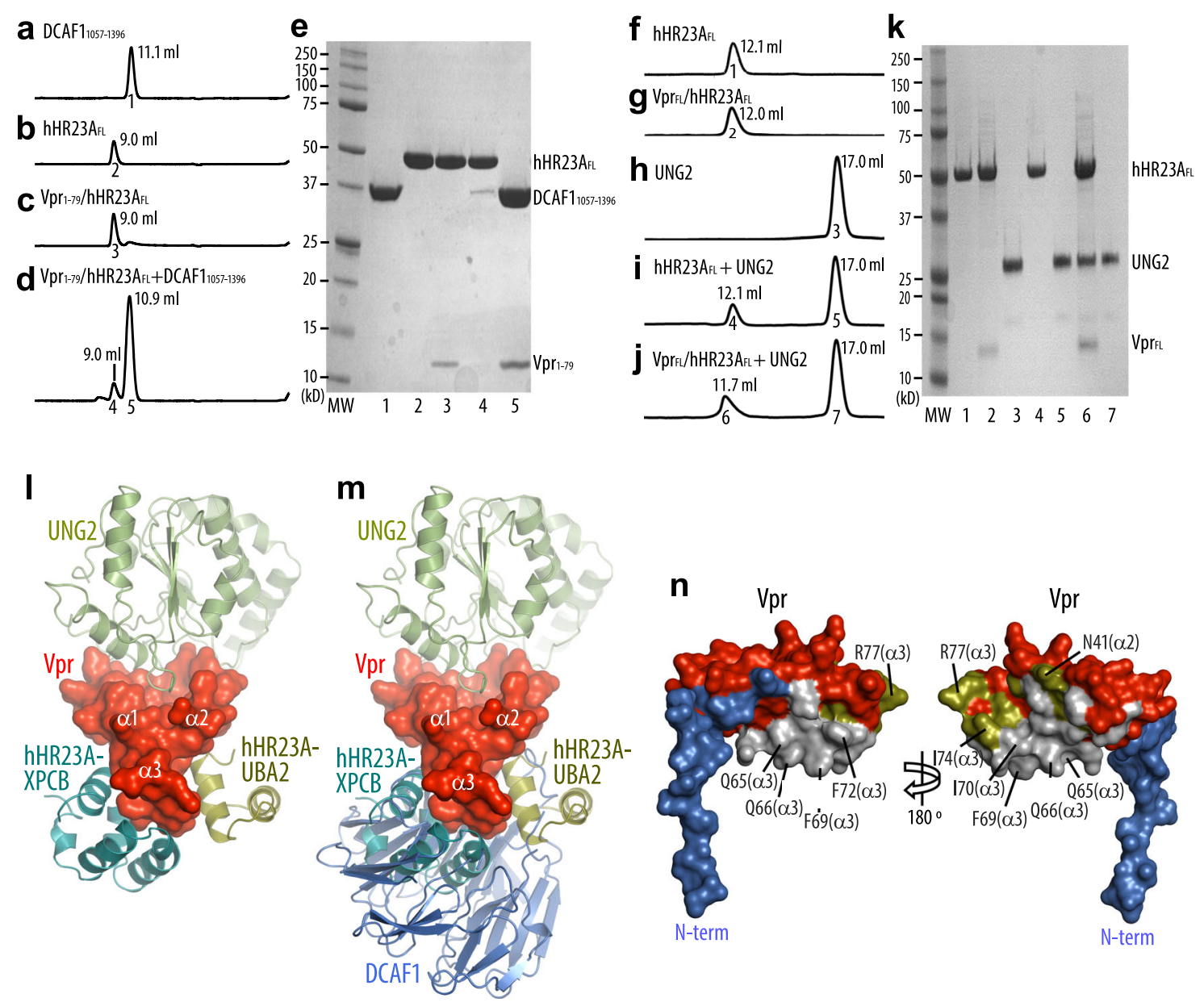

Fig. 5 hHR23A is displaced from the Vpr/hHR23A complex by DCAF1. Analytical gel-filtration chromatography elution profiles of a DCAF1 $1057-1396$, b hHR23A $\mathrm{FL}_{\mathrm{FL}} \mathbf{c}$ the $\mathrm{Vpr}_{1-79} / \mathrm{hHR} 23 \mathrm{~A}_{\mathrm{FL}}$ complex, $\mathbf{d}$ an equimolar mixture of the $\mathrm{Vpr}_{1-79} / \mathrm{hHR} 23 \mathrm{~A}_{\mathrm{FL}}$ complex and DCAF1 $1057-1396$ and $\mathbf{e}$ SDS-PAGE analysis of the elution peaks; Lane numbers correspond to peak numbers in (a-d). Analytical gel-filtration chromatography elution profiles of $\mathbf{f} h H R 23 \mathrm{~A}_{\mathrm{FL}}, \mathbf{g}$ the $\mathrm{Vpr}_{\mathrm{FL}} /$ hHR23A $A_{F L}$ complex, $\mathbf{h} U N G 2, \mathbf{i}$ an equimolar mixture of $h H R 23 A_{F L}$ and UNG2, $\mathbf{j}$ an equimolar mixture of the $V_{p r} r_{F L} / h H R 23 A_{F L}$ complex and UNG2 and $\mathbf{k}$ SDS-PAGE analysis of the elution peaks. Lane numbers correspond to peak numbers in (f-j). Two independent experiments were performed yielding

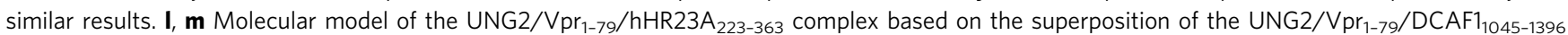
complex and the current $\mathrm{Vpr}_{1-79}-\mathrm{L}-\mathrm{hHR} 23 \mathrm{~A}_{223-363}$ complex without (I) and with displaying DCAF1 (m). $\mathbf{n}$ Surface representation of $\mathrm{Vpr}_{1-79}$ in two views,

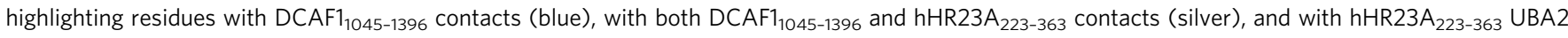
only contacts (olive). $\mathrm{Vpr}_{1-79}$ residues that do not interact with $\mathrm{DCAF1}_{1045-1396}$ or hHR23A $\mathrm{A}_{223-363}$ are shown in red.

of a multitude of additional substrate proteins tentatively identified by recent proteomic studies ${ }^{45}$. Together, these findings illustrate the remarkable versatility of HIV-1Vpr and reveal how it can interact specifically with a wide array of structurally dissimilar cellular targets.

Key residues involved in hHR23A binding are conserved in all HIV-1 Vpr isolates, yet absent in HIV-2 Vpr isolates (Sequences in the Los Alamos Data base; Supplementary Fig. 5e). This suggests that HIV-1 Vpr is uniquely tailored to engage hHR23A, consistent with a previous report ${ }^{46}$. It also contrasts with the observation that the DCAF1 interface residues are entirely conserved among HIV-1 and HIV-2 Vpr proteins. Since hijacking of CRL4 ${ }^{\text {DCAF1 }}$ E3 does not fully account for all known cellular effects of HIV-1 Vpr $25,26,46,47$, its interactions with hHR23A(B) are likely biologically relevant. Considering that $\mathrm{Vpr}$ can interact with both hHR23A and hHR23B, and both proteins appear to have largely overlapping functions, binding of $\mathrm{Vpr}$ may impact downstream pathways associated with both isoforms.

It is evident from our biochemical studies that $\mathrm{Vpr}$ associates with the XPC/hHR23A complex that plays key roles in postreplication DNA repair as well as transcription. Since Vpr binding to hHR23 does not require the presence of XPC in the complex, hHR23 probably acts as a bridge, linking Vpr with XPC. Although the atomic structure of the hHR23A/XPC complex, and in particular the detailed interaction between the XPCB domain of hHR23A and XPC, has not been elucidated yet, this possibility is consistent with predictions from the crystal structure of the homologous yeast $\operatorname{Rad} 23 / \operatorname{Rad} 4$ complex ${ }^{48}$. In this structure, the UBA2 domain of $\operatorname{Rad} 23$ makes only a few contacts with $\operatorname{Rad} 4$, leaving most of the UBA2 domain available for interactions with other proteins, e.g, Vpr (Supplementary Figs. 6 and 7). Although the relative orientation between the domains varies considerably, given the long flexible linker between them, it is nonetheless possible to achieve a satisfactory superposition without steric clashes by rotating the XPCB domain (Supplementary Figs. 6 and 7 ). Thus, both the homologous human and yeast complexes appear to share similar overall architecture that is predicted to allow for Vpr binding to hHR23A in the presence of XPC.

Although the exact role of the Vpr interaction with the hHR23/ XPC complex in HIV-1 biology is not yet known, possible scenarios are suggested by known functions of hHR23/XPC complexes and those of HIV-1 Vpr. In particular, the hHR23/XPC 
complex recognizes lesions that thermodynamically destabilize DNA duplexes in the early steps of post-replication nucleotide excision repair, including lesions resulting from oxidative DNA damage $^{29}$. HIV-1 reverse transcriptase is error prone, and Vpr has been shown to modulate HIV-1 mutation rates as well as other functional aspects of cellular post-replication DNA repair machinery ${ }^{49,50}$. The latter includes antagonizing select enzymes that mediate processing of branched DNA structures as well as

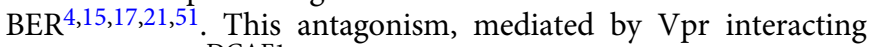
with the CRL4 ${ }^{\mathrm{DCAF}}$ E3 ligase, relieves restrictions imposed by these enzymes on HIV-1 replication ${ }^{4,22,52}$. In contrast, Vpr's interaction with hHR23/XPC does not involve DCAF1 and does not lead to its ubiquitination and degradation. Thus, it may well be that Vpr recruits the hHR23/XPC complex to facilitate some aspect(s) of proviral DNA repair. Intriguingly, our tentative conclusion that the interaction with hHR23 is specific to HIV-1 Vpr fits well with the previous finding of a more robust remodeling of the cellular DNA repair machinery by HIV-1, compared to HIV-2 $\mathrm{Vpr}^{19}$. This is also consistent with the model in which Vpr aids HIV-1 replication by modulating the DNA repair function of the hHR23/XPC complex. Of note, whereas HIV-1 $\mathrm{Vpr}$ is known to activate DNA damage checkpoint leading to cell cycle arrest in G2 phase, the Vpr-hHR23 interaction is not required for this function (Supplementary Figs. 8 and 9) consistent with previous reports ${ }^{25,26,46}$.

HIV-1 Vpr activates expression of HIV-1 both prior to integration and post integration. It, therefore, may also modulate expression of cellular genes ${ }^{53-55}$. Interestingly, hHR23/XPC is involved in transcriptional co-activation via histone acetylation and chromatin activation at a subset of RNA polymerase II promoters $^{31,32}$, thus suggesting another role for the Vpr/hHR23 interaction. It is conceivable that the recruitment of the hHR23/ XPC complex to active chromatin plays a role in transcriptional effects exerted by HIV-1 Vpr. Future studies are needed to address any possible involvement of the hHR23/XPC complex in the modulation of gene expression and cellular DNA repair processes by HIV-1 Vpr.

In summary, this study and previous structural and biochemical work suggest varied ways by which HIV-1 Vpr can coordinate specific interactions with a large number of structurally unrelated proteins. All lentiviral Vpr family proteins share their core structure with HIV-1 Vpr, yet exhibit extensive amino acid sequence variation on their surfaces. This sequence variability in conjunction with the molecular organization into a plastic threehelix bundle permits promiscuity in protein-protein interactions, providing a versatile means to interfere with multiple key cellular pathways.

\footnotetext{
Methods

Cloning and construction of plasmids. DNA coding for hHR23 $\mathrm{A}_{223-363}$ and $\mathrm{Vpr}_{1-79}-\mathrm{L}-\mathrm{hHR} 23 \mathrm{~A}_{223-363}$ was inserted into pET21 (EMD Chemicals).

hHR23A $A_{223-363}$ and NusA-Vpr ${ }_{1-79}$ gene constructs were inserted into a pET-DUET vector (EMD Chemicals) for co-expression. The $\mathrm{Vpr}_{1-79}-\mathrm{L}-\mathrm{hHR} 23 \mathrm{~A}_{223-363}$ construct contained a $\mathrm{His}_{6}$ tag $(\mathrm{LEHHHHHH})$ at its C-terminus. For mammalian cell expression, cDNAs encoding epitope-tagged full-length (FL) hHR23A, hHR23B, XPC and HIV-1 NL4-3 Vpr wild type and mutant alleles were cloned into pCG vector $^{56}$. All primers used are listed in Supplementary Table 3.
}

Protein expression and purification. $\mathrm{hHR} 23 \mathrm{~A}_{223-363}, \mathrm{hHR} 23 \mathrm{~A}_{\mathrm{FL}}, \operatorname{Trx}-\mathrm{hHR} 23 \mathrm{~A}_{\mathrm{FL}}$, NusA-Vpr $1-79$, UNG2, Vpr $_{1-79}-\mathrm{L}-\mathrm{hHR} 23 \mathrm{~A}_{223-363}, \mathrm{hHR} 23 \mathrm{~A}_{223-363}-\mathrm{L}_{-\mathrm{Vpr}_{1-79}}$ $\mathrm{Vpr}_{1-79} / \mathrm{hHR} 23 \mathrm{~A}_{223-363}, \mathrm{Vpr}_{1-79} / \mathrm{hHR} 23 \mathrm{~A}_{\mathrm{FL}}$ and $\mathrm{Vpr}_{\mathrm{FL}} / \mathrm{hHR} 23 \mathrm{~A}_{\mathrm{FL}}$ complexes were expressed or co-expressed in E. coli Rosetta 2 (DE3) or Rosetta 2 (DE3) pLysS cells (EMD Chemicals) in the presence of $0.1 \mathrm{mM} \mathrm{ZnCl}_{2}$, using $0.5 \mathrm{mM}$ isopropyl 1-thio$\beta$-D-galactopyranoside for induction over $16 \mathrm{~h}$ at $18^{\circ} \mathrm{C}$. Uniform ${ }^{2} \mathrm{H}-,{ }^{15} \mathrm{~N}-$, and ${ }^{13} \mathrm{C}$ - labeling of the proteins was achieved using modified minimal medium, containing ${ }^{2} \mathrm{H}_{2} \mathrm{O},{ }^{15} \mathrm{NH}_{4} \mathrm{Cl}$ and ${ }^{13} \mathrm{C}_{6}$-glucose as deuterium, nitrogen and carbon sources, while unlabeled proteins were prepared in Luria-Bertani (LB) medium. Cells were harvested by centrifugation at $6000 \times g$ and re-suspended in lysis buffer, containing $50 \mathrm{mM}$ sodium phosphate $(\mathrm{pH} 7.5), 10 \mathrm{mM}$ imidazole and $200 \mathrm{mM}$
$\mathrm{NaCl}$. Cells were lysed using a microfluidizer (Microfluidics, MA) or, for hHR23A $223-363$ and $\mathrm{Vpr}_{1-79}-\mathrm{L}-\mathrm{hHR} 23 \mathrm{~A}_{223-363}$ samples, by sonication (Sonicator 3000; Misonix, Farmingdale, NY). The lysed cells were centrifuged at 40,000 $\times g$, and the supernatant was applied to a 5 -ml HisTrap (GE healthcare) $\mathrm{His}_{6}$-tag affinity column, equilibrated in lysis buffer. Bound proteins were eluted using a linear gradient of $0-0.5 \mathrm{M}$ imidazole. Protein containing fractions were further purified over a HiPrep Superdex 75 or HiPrep Superdex $200(2.6 \mathrm{~cm} \times 60 \mathrm{~cm}$, GE healthcare $)$ gel filtration column, equilibrated in $25 \mathrm{mM}$ sodium phosphate buffer ( $\mathrm{pH} 7.5$ ), $100 \mathrm{mM} \mathrm{NaCl}, 2 \mathrm{mM}$ DTT and $0.02 \%$ sodium azide. $\mathrm{Vpr}_{1-79}-\mathrm{L}-\mathrm{hHR} 23 \mathrm{~A}_{223-363}$, $\mathrm{Vpr}_{1-79} / \mathrm{hHR} 23 \mathrm{~A}_{223-363}, \mathrm{Vpr}_{1-79} / \mathrm{hHR} 23 \mathrm{~A}_{\mathrm{FL}}$ and $\mathrm{Vpr}_{\mathrm{FL}} / \mathrm{hHR} 23 \mathrm{~A}_{\mathrm{FL}}$ complexes were further purified over a HiTrap Q column, equilibrated in $25 \mathrm{mM}$ sodium phosphate buffer ( $\mathrm{pH} 7.5$ ), $2 \mathrm{mM}$ DTT and $0.02 \%$ sodium azide. Bound proteins were eluted using a linear gradient of $0-1 \mathrm{M} \mathrm{NaCl}$. Protein fractions were pooled, concentrated for crystallization or buffer-exchanged in Amicon Ultra concentrators (Millipore) for NMR studies against NMR buffers: 10 mM MES (pH 6.5), 10 mM HEPES (pH 7.2 or 7.5 ) or $25 \mathrm{mM}$ sodium phosphate ( $\mathrm{pH} 7.2$ or 7.5 ), each containing $50 \mathrm{mM}$ $\mathrm{NaCl}, 2-5 \mathrm{mM}$ DTT or $1 \mathrm{mM}$ TCEP, $0.1 \mathrm{mM} \mathrm{ZnSO}_{4}, 0.1 \mathrm{mM}$ EDTA and $0.02 \%$ sodium azide. The correct molecular masses of proteins were confirmed by LC-ESI-TOF mass spectrometry (Bruker Daltonics, Billerica, MA). DCAF $1157-1396$ was expressed in sf9 insect cells and was purified using a $5 \mathrm{ml}$ HisTrap (GE healthcare $)$ and HiPrep Superdex200 $(2.6 \mathrm{~cm} \times 60 \mathrm{~cm}$, GE healthcare $)$ gel filtration column as described above.

\section{Crystallization, structure determination and refinement. Crystals for} $\mathrm{Vpr}_{1-79}-\mathrm{L}-\mathrm{hHR} 23 \mathrm{~A}_{223-363}$ were grown at $16^{\circ} \mathrm{C}$ by hanging drop vapor diffusion, using a mixture of $0.5 \mu \mathrm{l}$ protein $(25 \mathrm{mg} / \mathrm{ml})$ with $0.5 \mu \mathrm{l}$ crystallization buffer $(0.1 \mathrm{M}$ Tris, pH 7.5, 10\% PEG 4000). Initial crystals were improved by streak seeding. Crystals were cryoprotected by the addition of $35 \%(\mathrm{v} / \mathrm{v})$ glycerol. Crystals belonging to space group $\mathrm{P} 3_{1} 21$, diffracting to $2.2 \AA$, were used for structure determination. Data were collected at the 9-2 SSRL (wavelength $0.979 \AA$, temperature $100 \mathrm{~K}$ ). Diffraction data were processed, integrated and scaled using $\mathrm{XDS}^{57}$. The structure was solved by de novo $\mathrm{Zn}^{2+}$ single-wavelength anomalous dispersion (Zn-SAD) phasing. Models were refined using the program BUSTER ${ }^{41}$ followed by several cycles of manual model building and $B$ factor sharpening in $\operatorname{Coot}^{43}$. Crystallographic statistics are summarized in Table 1.

NMR spectroscopy. NMR data were collected on Bruker 600, 700, 800, and $900 \mathrm{MHz}$ AVANCE spectrometers. All spectrometers were equipped with $z$-axis gradient, triple resonance cryoprobes. Experiments were performed at $298 \mathrm{~K}$. A homonuclear 2D NOESY spectrum was acquired on unlabeled $\mathrm{Vpr}_{1-79}-\mathrm{L}$ hHR23A $223-363$. Heteronuclear 2D ${ }^{1} \mathrm{H}_{-}{ }^{15} \mathrm{~N}$ HSQC and ${ }^{1} \mathrm{H}-{ }^{13} \mathrm{C}$ HSQC and 3D HNCACB, HN(CO)CACB, HNCA, HN(CO)CA, HBHACONH, HCCCONH, CCCONH, HCCH-TOCSY (mixing time $10.9 \mathrm{~ms}$ ) and simultaneously ${ }^{13} \mathrm{C} /{ }^{15} \mathrm{~N}$-edited NOESY (mixing time $100 \mathrm{~ms}$ ) experiments were performed using either $15 \mathrm{~N}$ - or 15N,13C-labeled Vpr1-79-L-hHR23A223-363, hHR23A223-363-L-Vpr1-79 (only 2D 1H-15N HSQC) and hHR23A223-363 samples. For ${ }^{2} \mathrm{H},{ }^{15} \mathrm{~N},{ }^{13} \mathrm{C}$-labeled $\mathrm{Vpr}_{1-79}-\mathrm{L}-\mathrm{hHR} 23 \mathrm{~A}_{223-363}$, 3D TROSY-type HNCACB, HN(CO)CACB spectra were also recorded. All data were processed with NMRPipe $e^{58}$ and TopSpin 3.1 (Bruker), and analyzed with $\mathrm{CCPN}^{59}$.

NMR structure determination. Structures were calculated in XPLOR-NIH ${ }^{60,61}$. An iterative approach with extensive manual cross-checking of all distance restraints against the NOESY data and the initial structures was employed using $\mathrm{CCPN}^{59}$. The final number of NOE-derived distance restraints is 7650 , of which 338 are intermolecular (81 $\mathrm{Vpr}_{1-79} / \mathrm{XPCB}$ domain and $257 \mathrm{Vpr}_{1-79} / \mathrm{UBA} 2$ domain), supplemented by $330 \phi$ and $\psi$ backbone torsion angles from TALOS ${ }^{62}$. In addition, a $\mathrm{Zn}^{2+}$ ion with tetrahedral coordination to the H33, H71, C76 and H78 side chains of $\mathrm{Vpr}_{1-79}$ was added at a late stage in the calculations, using distances from the X-ray structure of the $\mathrm{Vpr}_{1-79} / \mathrm{UBA} 2$ domain. In total, 960 structures were generated, and the 55 lowest energy structures were selected and analyzed using XPLOR-NIH ${ }^{63}$ and PROCHECK-NMR ${ }^{64}$. All structure figures except Supplementary Fig. 1e were generated with Pymol. Supplementary Fig. 1e was generated with MOLMOL ${ }^{65}$.

In-gel trypsin digestion for mass spectrometry. Gel bands containing (1) a $\mathrm{Vpr}_{1-79}-\mathrm{L}-\mathrm{hHR} 23 \mathrm{~A}_{223-363}$ solution employed to set up crystallization trays (control) or (2) dissolved crystals of $\mathrm{Vpr}_{1-79}-\mathrm{L}-\mathrm{hHR} 23 \mathrm{~A}_{223-363}$ were excised and proteins were digested with trypsin ${ }^{66}$. Briefly, gel bands were diced into small pieces $\left(<1 \mathrm{~mm}^{3}\right)$ and washed with a solution of $50 \%$ acetonitrile $(\mathrm{ACN}) / 25 \mathrm{mM}$ ammonium bicarbonate until no more visible stain was present. The gel pieces were then dehydrated with $100 \% \mathrm{ACN}$, reduced with $10 \mathrm{mM}$ DTT at $56^{\circ} \mathrm{C}$ for $1 \mathrm{~h}$, followed by alkylation with $55 \mathrm{mM}$ Iodoacetamide (IAA) at room temperature for $45 \mathrm{~min}$ in the dark. Excess DTT and IAA were removed by washing the gel pieces with $25 \mathrm{mM}$ ammonium bicarbonate and then twice with $100 \%$ ACN. A solution containing $20 \mathrm{ng} / \mu \mathrm{L}$ sequencing grade modified trypsin (Promega Corporation, Madison, WI; catalog\#V511A) and $25 \mathrm{mM}$ ammonium bicarbonate was added to cover the gel pieces and digestion was carried out overnight at $37^{\circ} \mathrm{C}$. The resultant tryptic peptides were extracted from the gel with $70 \% \mathrm{ACN} / 5 \%$ formic acid (FA), 
vacuum dried, and reconstituted in $18 \mu \mathrm{L} 0.1 \%$ FA for nanoflow liquidchromatography tandem mass spectrometry (nLC-MS/MS analysis).

Tandem mass spectrometry. Tryptic peptides were analyzed by nLC-MS/MS using a NanoAcquity UPLC (Waters' Corporation, Milford, MA) interfaced to a Velos Pro linear ion trap mass spectrometer (Thermo Fisher Scientific, Waltham, MA, USA). For each analysis, a $1 \mu \mathrm{L}$ volume of protein digest was injected onto a C18 column (PicoChip ${ }^{\text {ts }}$ column packed with Reprosil C18 $3 \mu \mathrm{m} 120 \AA$ chromatography media in a $10.5 \mathrm{~cm}$ long, $75 \mu \mathrm{m}$ ID column with a $15 \mu \mathrm{m}$ tip, New Objective, Inc., Woburn, MA, USA) and eluted off to the mass spectrometer using a $37-\mathrm{min}$ linear gradient of $3-35 \% \mathrm{ACN} / 0.1 \% \mathrm{FA}$ at a flow rate of $300 \mathrm{~nL} / \mathrm{min}$. The Velos Pro was operated in positive ionization mode with a spray voltage of $1.95 \mathrm{kV}$ and capillary temperature of $275^{\circ} \mathrm{C}$. Acquisition consisted of cycles of one full-scan MS1 (AGC of $3 \times 10^{4}, 75 \mathrm{~ms}$ maximum ion accumulation time, and $\mathrm{m} / \mathrm{z}$ range of 375-1800) followed by eight MS/MS spectra recorded sequentially for the most abundant ions in the ion trap (minimum signal required 1000 counts, $1 \times 10^{4}$ AGC target, $100 \mathrm{~ms}$ maximum injection time, isolation width $2 \mathrm{~m} / z$, normalized collision energy 35 , and activation time $10 \mathrm{~ms}$ ). Dynamic exclusion $(30 \mathrm{~s})$ was enabled to minimize redundant selection of peptides previously selected for MS/MS.

Mass spectrometry data analysis. Collected MS/MS spectra were searched using the MASCOT search engine v2.4.0 (Matrix Science Ltd., London, England) ${ }^{67}$ against the UniProt E. coli database, downloaded on September 5, 2014, with 5803 entries, plus the sequence for $23 \mathrm{~A} 2 \mathrm{~V}$, using the following modifications: static modification of cysteine carbamidomethylation $(+57)$, variable modifications of methionine oxidation $(+16)$, and protein $\mathrm{N}$-terminal acetylation $(+42)$. The mass tolerance was set to 1.4 Da for the precursor ions and 0.8 Da for the fragment ions. Peptide identifications were filtered using the PeptideProphet ${ }^{\mathrm{TM}}$ and ProteinProphet ${ }^{\circledast}$ algorithms with a protein threshold cutoff of $99 \%$, minimum of 2 peptides, and peptide threshold cutofff of $90 \%$ implemented in Scaffold ${ }^{\mathrm{TM}}$ v4.11.0 (Proteome software, Portland, OR).

GST pulldown assays. $1000 \mathrm{pmol}$ of GST-tagged WT hHR23A/B or mutant proteins were incubated with 1000 pmol NusA-Vpr $\mathrm{FL}_{\mathrm{F}}$ in $500 \mu \mathrm{l}$ PBS buffer (binding buffer) for $30 \mathrm{~min}$ at $4{ }^{\circ} \mathrm{C}$. TEV cleavage $(50 \mathrm{pmol})$ was carried out at $4{ }^{\circ} \mathrm{C}$ overnight. After centrifugation at $21,000 \times g$ for $20 \mathrm{~min}$ (to remove precipitated, excess Vpr) Glutathion-Sepharose $4 \mathrm{~B}$ beads ( $50 \mu \mathrm{l}$ of a $50 \%$ slurry) were added to the supernatant for $1 \mathrm{~h}$ at $4{ }^{\circ} \mathrm{C}$. The beads were washed four times with $1 \mathrm{ml}$ of binding buffer, followed by addition of $50 \mu \mathrm{l}$ SDS-PAGE loading buffer. Proteins were separated by $4-20 \%$ SDS-PAGE, transferred to PVDF membrane, and detected by immunoblotting with an anti-His antibody (1:1000 dilution, cat. \#05949, clone His.H8, Millipore).

Transfections, immunoprecipitations and immunoblotting. Transfections of HEK 293T cells were performed using the calcium phosphate co-precipitation method $^{68}$. Whole-cell extracts were immunoprecipitated with FLAG-M2 beads (Sigma-Aldrich). Immune complexes were eluted by competition with FLAGpeptide under native conditions, separated by SDS-PAGE and transferred to PVDF membranes for immunoblotting. Proteins were detected with antibodies specific for hHR23B (1:1000 dilution, cat. \#13525, clone D4W7F, Cell Signaling Technology), FLAG- (1:1000, cat. \#F1804, clone M2, Sigma), HA- (1:2000, 12CA5, PMID: 6204768) or myc- (1:1000, 9E10, PMID: 3915782) epitope tags, immune complexes revealed with fluorescent secondary antibodies specific for the mouse $(1: 10,000$, IRDye 800CW Gt anti-mouse IgG Li-Cor cat. \#926-322100, or rabbit IgG (IRDye 680RD Gt anti-rabbit IgG 1:10,000, Li-Cor cat. \#926-68071) and fluorescent signals quantified using Li-Cor Odyssey imager and software version 3.0.30 (Li-Cor).

Cell cycle analysis. HEK293T cells were seeded in 12-well plates and cotransfected with plasmids expressing HIV-1 FLAG-tagged HIV-1 Vpr.wt or mutant proteins and GFP marker protein. $48 \mathrm{~h}$ post transfection, the cells were trypsinized, washed twice with PBS, fixed with $70 \%$ ethanol overnight, washed twice with PBS and stained with propidium iodide. GFP and propidium iodide fluorescence of the cells was analyzed by flow cytometry (BD LSRII). Data were analyzed using FlowJo software (Tree Star Inc., Ashland, OR). One-way ANOVA analysis was performed within GraphPad Prism 9.

Analytical gel filtration column chromatography. One hundred $\mu \mathrm{l}$ of proteins at $20 \mu \mathrm{M}$ (hHR23A $\mathrm{AL}_{\mathrm{FL}}, \mathrm{Vpr}_{\mathrm{FL}} / \mathrm{hHR} 23 \mathrm{~A}_{\mathrm{FL}}$ complex, UNG2, an equimolar mixture of $\mathrm{hH} 23 \mathrm{~A}_{\mathrm{FL}}$ and UNG2 or $\mathrm{Vpr}_{\mathrm{FL}} / \mathrm{hHR} 23 \mathrm{~A}_{\mathrm{FL}}$ complex and UNG2) or $60 \mu$ of proteins at $21 \mu \mathrm{M}\left(\mathrm{DCAF}_{1057-1396}, \mathrm{hHR} 23 \mathrm{~A}_{\mathrm{FL}}, \mathrm{Vpr}_{1-79} / \mathrm{hHR} 23 \mathrm{~A}_{\mathrm{FL}}\right.$ complex or an equimolar mixture of $\mathrm{Vpr}_{1-79} / \mathrm{hHR} 23 \mathrm{~A}_{\mathrm{FL}}$ complex and DCAF1 $1_{1057-1396}$ ) was injected onto a $24-\mathrm{ml}$ analytical Superdex75 column (equilibrated in $25 \mathrm{mM}$ sodium phosphate, $150 \mathrm{mM} \mathrm{NaCl}, 5 \%$ glycerol, $1 \mathrm{mM} \mathrm{DTT}$ and $0.02 \% \mathrm{NaN}_{3}$ ) at a flow rate of $0.5 \mathrm{ml} / \mathrm{min}$. Each peak was concentrated and analyzed by SDS-PAGE with Coomassie Blue staining.

Isothermal titration calorimetry (ITC). Experiments were performed using a MicroCal PEAQ-ITC calorimeter (Malvern). Proteins were dialyzed overnight against $20 \mathrm{mM}$ sodium phosphate buffer, pH 7.4, $100 \mathrm{mM} \mathrm{NaCl}$ and concentrated in an Amicon concentrator (EMD Millipore). NusA-Vpr ${ }_{1-79}$ protein $(8.5 \mu \mathrm{M})$ was placed in the sample cell and either Trx-hHR23A $A_{F L}$ or DCAF $1_{1045-1396} / D D B 1$ proteins $(100 \mu \mathrm{M})$ were added in aliquots at $18^{\circ} \mathrm{C}$. Data analyses were performed with the PEAQ-ITC analysis software.

Reporting summary. Further information on research design is available in the Nature Research Reporting Summary linked to this article.

\section{Data availability}

The coordinates of the crystal structure of the $\mathrm{Vpr}_{1-79} / \mathrm{hHR} 23 \mathrm{~A}-\mathrm{UBA} 2$ complex have been deposited to the Protein Data Bank under accession number PDB 6XQI. The coordinates of the NMR structures of the $\mathrm{Vpr}_{1-79}-\mathrm{L}-\mathrm{hHR} 23 \mathrm{~A}_{223-363}$ complex have been deposited to the Protein Data Bank under accession number PDB 6XQJ, and the NMR assignments of the complex have been deposited to the Biological Magnetic Resonance Bank (https://bmrb.io/) under entry number 30769, which is available at https://doi.org/ 10.13018/BMR30769. Previously published PDB accession codes that have been used in this study are 5JK7 (crystal structure of the DDB1/DCAF1/Vpr/UNG2 complex), 2QSF (crystal structure of the yeast Rad23/Rad4 complex), and 4UN2 (crystal structure of the UBA domain of Dsk2 in complex with ubiquitin). Source data are provided with this manuscript.

Received: 1 July 2020; Accepted: 26 October 2021;

Published online: 25 November 2021

\section{References}

1. Sauter, D. \& Kirchhoff, F. Multilayered and versatile inhibition of cellular antiviral factors by HIV and SIV accessory proteins. Cytokine Growth Factor Rev. 40, 3-12 (2018).

2. Herate, C. et al. Uracil DNA glycosylase interacts with the p32 subunit of the replication protein A complex to modulate HIV-1 reverse transcription for optimal virus dissemination. Retrovirology 13, 26 (2016).

3. Vermeire, J. et al. HIV triggers a cGAS-dependent, Vpu- and Vpr-regulated Type I Interferon response in CD4(+) T cells. Cell Rep. 17, 413-424 (2016).

4. Yan, J., Shun, M. C., Zhang, Y., Hao, C. \& Skowronski, J. HIV-1 Vpr counteracts HLTF-mediated restriction of HIV-1 infection in T cells. Proc. Natl Acad. Sci. USA 116, 9568-9577 (2019).

5. Connor, R. I., Chen, B. K., Choe, S. \& Landau, N. R. Vpr is required for efficient replication of human immunodeficiency virus type-1 in mononuclear phagocytes. Virology 206, 935-944 (1995).

6. Malim, M. H. \& Emerman, M. HIV-1 accessory proteins-ensuring viral survival in a hostile environment. Cell Host Microbe 3, 388-398 (2008).

7. Collins, D. R. \& Collins, K. L. HIV-1 accessory proteins adapt cellular adaptors to facilitate immune evasion. PLoS Pathog. 10, e1003851 (2014).

8. Tristem, M., Purvis, A. \& Quicke, D. L. Complex evolutionary history of primate lentiviral vpr genes. Virology 240, 232-237 (1998).

9. Sharp, P. M., Bailes, E., Stevenson, M., Emerman, M. \& Hahn, B. H. Gene acquisition in HIV and SIV. Nature 383, 586-587 (1996).

10. Peeters, M. \& Courgnaud, V. Overview of primate lentiviruses and their evolution in non-human primates in Africa. In HIV Sequence Compendium (eds Kuiken, C. et al.) 2-23 (Theoretical Biology and Biophysics Group, Los Alamos National Laboratory, Los Alamos, NM, 2002).

11. Le Rouzic, E. et al. HIV1 Vpr arrests the cell cycle by recruiting DCAF1/ VprBP, a receptor of the Cul4-DDB1 ubiquitin ligase. Cell Cycle 6, 182-188 (2007).

12. Romani, B. \& Cohen, E. A. Lentivirus Vpr and Vpx accessory proteins usurp the cullin4-DDB1 (DCAF1) E3 ubiquitin ligase. Curr. Opin. Virol. 2, 755-763 (2012).

13. Hrecka, K. et al. Lentiviral Vpr usurps Cul4-DDB1[VprBP] E3 ubiquitin ligase to modulate cell cycle. Proc. Natl Acad. Sci. USA 104, 11778-11783 (2007).

14. Selig, L. et al. Uracil DNA glycosylase specifically interacts with Vpr of both human immunodeficiency virus type 1 and simian immunodeficiency virus of sooty mangabeys, but binding does not correlate with cell cycle arrest. J. Virol. 71, 4842-4846 (1997).

15. Ahn, J. et al. HIV-1 Vpr loads uracil DNA glycosylase-2 onto DCAF1, a substrate recognition subunit of a cullin 4A-ring E3 ubiquitin ligase for proteasome-dependent degradation. J. Biol. Chem. 285, 37333-37341 (2010).

16. Laguette, N. et al. Premature activation of the SLX4 complex by Vpr promotes G2/M arrest and escape from innate immune sensing. Cell 156, 134-145 (2014).

17. Zhou, X., DeLucia, M. \& Ahn, J. SLX4-SLX1 protein-independent downregulation of MUS81-EME1 protein by HIV-1 viral protein R (Vpr). J. Biol. Chem. 291, 16936-16947 (2016)

18. Lahouassa, H. et al. HIV-1 Vpr degrades the HLTF DNA translocase in T cells and macrophages. Proc. Natl Acad. Sci. USA 113, 5311-5316 (2016). 
19. Hrecka, K. et al. HIV-1 and HIV-2 exhibit divergent interactions with HLTF and UNG2 DNA repair proteins. Proc. Natl Acad. Sci. USA 113, E3921-E3930 (2016).

20. Zhou, X. et al. HIV-1 Vpr protein directly loads helicase-like transcription factor (HLTF) onto the CRL4-DCAF1 E3 ubiquitin ligase. J. Biol. Chem. 292, 21117-21127 (2017).

21. Yan, J. et al. HIV-1 Vpr reprograms CLR4(DCAF1) E3 ubiquitin ligase to antagonize exonuclease 1-mediated restriction of HIV-1 infection. mBio $\mathbf{9}$, e01732-18 (2018).

22. Lv, L. et al. Vpr targets TET2 for degradation by CRL4(VprBP) E3 ligase to sustain IL-6 expression and enhance HIV-1 replication. Mol. Cell 70, 961-970e5 (2018).

23. Wu, Y. et al. The DDB1-DCAF1-Vpr-UNG2 crystal structure reveals how HIV-1 Vpr steers human UNG2 toward destruction. Nat. Struct. Mol. Biol. 23, 933-940 (2016).

24. Hansen, E. C. et al. Diverse fates of uracilated HIV-1 DNA during infection of myeloid lineage cells. Elife 5, e18447 (2016).

25. Withers-Ward, E. S. et al. Human immunodeficiency virus type $1 \mathrm{Vpr}$ interacts with HHR23A, a cellular protein implicated in nucleotide excision DNA repair. J. Virol. 71, 9732-9742 (1997).

26. Gragerov, A., Kino, T., Ilyina-Gragerova, G., Chrousos, G. P. \& Pavlakis, G. N. HHR23A, the human homologue of the yeast repair protein RAD23, interacts specifically with Vpr protein and prevents cell cycle arrest but not the transcriptional effects of Vpr. Virology 245, 323-330 (1998).

27. Withers-Ward, E. S., Mueller, T. D., Chen, I. S. \& Feigon, J. Biochemical and structural analysis of the interaction between the UBA(2) domain of the DNA repair protein HHR23A and HIV-1 Vpr. Biochemistry 39, 14103-14112 (2000).

28. Yokoi, M. \& Hanaoka, F. Two mammalian homologs of yeast Rad23, HR23A and HR23B, as multifunctional proteins. Gene 597, 1-9 (2017).

29. Sugasawa, K. Mechanism and regulation of DNA damage recognition in mammalian nucleotide excision repair. Enzymes 45, 99-138 (2019).

30. Cattoglio, C. et al. Functional and mechanistic studies of XPC DNA-repair complex as transcriptional coactivator in embryonic stem cells. Proc. Natl Acad. Sci. USA 112, E2317-E2326 (2015).

31. Bidon, B. et al. XPC is an RNA polymerase II cofactor recruiting ATAC to promoters by interacting with E2F1. Nat. Commun. 9, 2610 (2018).

32. Semer, M. et al. DNA repair complex licenses acetylation of H2A.Z.1 by KAT2A during transcription. Nat. Chem. Biol. 15, 992-1000 (2019).

33. Schauber, C. et al. Rad23 links DNA repair to the ubiquitin/proteasome pathway. Nature 391, 715-718 (1998).

34. Shi, Y. et al. Rpn1 provides adjacent receptor sites for substrate binding and deubiquitination by the proteasome. Science 351, 831-841 (2016).

35. Ortolan, T. G. et al. The DNA repair protein rad23 is a negative regulator of multi-ubiquitin chain assembly. Nat. Cell Biol. 2, 601-608 (2000).

36. Morellet, N., Bouaziz, S., Petitjean, P. \& Roques, B. P. NMR structure of the HIV-1 regulatory protein VPR. J. Mol. Biol. 327, 215-227 (2003).

37. Jung, J. et al. Binding of HIV-1 Vpr protein to the human homolog of the yeast DNA repair protein RAD23 (hHR23A) requires its xeroderma pigmentosum complementation group $\mathrm{C}$ binding (XPCB) domain as well as the ubiquitin-associated 2 (UBA2) domain. J. Biol. Chem. 289, 2577-2588 (2014).

38. Dieckmann, T. et al. Structure of a human DNA repair protein UBA domain that interacts with HIV-1 Vpr. Nat. Struct. Biol. 5, 1042-1047 (1998).

39. Walters, K. J., Lech, P. J., Goh, A. M., Wang, Q. \& Howley, P. M. DNA-repair protein hHR23a alters its protein structure upon binding proteasomal subunit S5a. Proc. Natl Acad. Sci. USA 100, 12694-12699 (2003).

40. Kamionka, M. \& Feigon, J. Structure of the XPC binding domain of hHR23A reveals hydrophobic patches for protein interaction. Protein Sci. 13, 2370-2377 (2004).

41. Blanc, E. et al. Refinement of severely incomplete structures with maximum likelihood in BUSTER-TNT. Acta Crystallogr. D 60, 2210-2221 (2004).

42. Smart, O. S. et al. Exploiting structure similarity in refinement: automated NCS and target-structure restraints in BUSTER. Acta Crystallogr. D 68, 368-380 (2012).

43. Emsley, P. \& Cowtan, K. Coot: model-building tools for molecular graphics. Acta Crystallogr. D 60, 2126-2132 (2004).

44. Di Marzio, P., Choe, S., Ebright, M., Knoblauch, R. \& Landau, N. R. Mutational analysis of cell cycle arrest, nuclear localization and virion packaging of human immunodeficiency virus type $1 \mathrm{Vpr}$. J. Virol. 69, 7909-7916 (1995).

45. Greenwood, E. J. D. et al. Promiscuous targeting of cellular proteins by Vpr drives systems-level proteomic remodeling in HIV-1 infection. Cell Rep. 27, 1579-1596 e7 (2019).

46. Mansky, L. M. et al. Interaction of human immunodeficiency virus type $1 \mathrm{Vpr}$ with the HHR23A DNA repair protein does not correlate with multiple biological functions of Vpr. Virology 282, 176-185 (2001).

47. Zhu, Q., Wani, G., Wani, M. A. \& Wani, A. A. Human homologue of yeast Rad23 protein A interacts with $\mathrm{p} 300 /$ cyclic AMP-responsive element binding
(CREB)-binding protein to down-regulate transcriptional activity of p53. Cancer Res. 61, 64-70 (2001).

48. Min, J. H. \& Pavletich, N. P. Recognition of DNA damage by the Rad4 nucleotide excision repair protein. Nature 449, 570-575 (2007).

49. Mansky, L. M. The mutation rate of human immunodeficiency virus type 1 is influenced by the vpr gene. Virology 222, 391-400 (1996).

50. Hu, W. S. \& Hughes, S. H. HIV-1 reverse transcription. Cold Spring Harb. Perspect. Med. 2, a006882 (2012).

51. Bouhamdan, M. et al. Human immunodeficiency virus type $1 \mathrm{Vpr}$ protein binds to the uracil DNA glycosylase DNA repair enzyme. J. Virol. 70, 697-704 (1996).

52. Weil, A. F. et al. Uracil DNA glycosylase initiates degradation of HIV-1 cDNA containing misincorporated dUTP and prevents viral integration. Proc. Natl Acad. Sci. USA 110, E448-E457 (2013).

53. Poon, B. \& Chen, I. S. Human immunodeficiency virus type 1 (HIV-1) Vpr enhances expression from unintegrated HIV-1 DNA. J. Virol. 77, 3962-3972 (2003).

54. Cohen, E. A. et al. Identification of HIV-1 vpr product and function. J. Acquir. Immune Defic. Syndr. (1988) 3, 11-18 (1990).

55. Kino, T. et al. The HIV-1 virion-associated protein vpr is a coactivator of the human glucocorticoid receptor. J. Exp. Med. 189, 51-62 (1999).

56. Tanaka, M. \& Herr, W. Differential transcriptional activation by Oct-1 and Oct-2: interdependent activation domains induce Oct-2 phosphorylation. Cell 60, 375-386 (1990).

57. Kabsch, W. Xds. Acta Crystallogr. D 66, 125-132 (2010).

58. Delaglio, F. et al. NMRPipe: a multidimensional spectral processing system based on UNIX pipes. J. Biomol. NMR 6, 277-293 (1995).

59. Vranken, W. F. et al. The CCPN data model for NMR spectroscopy: development of a software pipeline. Proteins 59, 687-696 (2005)

60. Schwieters, C. D., Kuszewski, J. J., Tjandra, N. \& Clore, G. M. The Xplor-NIH NMR molecular structure determination package. J. Magn. Reson. 160, 65-73 (2003).

61. Schwieters, C. D., Kuszewski, J. J. \& Marius Clore, G. Using Xplor-NIH for NMR molecular structure determination. Prog. Nucl. Magn. Reson. Spectrosc. 48, 47-62 (2006).

62. Cornilescu, G., Delaglio, F. \& Bax, A. Protein backbone angle restraints from searching a database for chemical shift and sequence homology. J. Biomol. NMR 13, 289-302 (1999).

63. Brunger, A. T. et al. Crystallography \& NMR system: a new software suite for macromolecular structure determination. Acta Crystallogr. D 54, 905-921 (1998).

64. Laskowski, R. A., Rullmannn, J. A., MacArthur, M. W., Kaptein, R. \& Thornton, J. M. AQUA and PROCHECK-NMR: programs for checking the quality of protein structures solved by NMR. J. Biomol. NMR 8, 477-486 (1996).

65. Koradi, R., Billeter, M. \& Wuthrich, K. MOLMOL: a program for display and analysis of macromolecular structures. J. Mol. Graph. 14, 29-32 (1996). 51-5.

66. Braganza, A. et al. UBE3B is a calmodulin-regulated, mitochondrionassociated E3 ubiquitin ligase. J. Biol. Chem. 292, 2470-2484 (2017).

67. Perkins, D. N., Pappin, D. J., Creasy, D. M. \& Cottrell, J. S. Probability-based protein identification by searching sequence databases using mass spectrometry data. Electrophoresis 20, 3551-3567 (1999).

68. Graham, F. L. \& van der Eb, A. J. A new technique for the assay of infectivity of human adenovirus 5 DNA. Virology 52, 456-467 (1973).

\section{Acknowledgements}

The authors thank Teresa Brosenitsch for critical reading of the manuscript, Doug Bevan for computer, Michael J. Delk for NMR, Doowon Lee for in-house X-ray technical support, Cynthia Hale for setting up quantitative Western blotting, and Dewayne Falkner and Ailing Liu for assistance with the cell cycle assay experiments. We also thank Michael Becker and Craig Ogata at GM/CA (Argonne National Laboratory) for user support and Aina Cohen, Irimpan Mathews, Clyde Smith, and Ana Gonzalez at Stanford Synchrotron Radiation Lightsource (SSRL) for assistance during data collection. The ITC instrument was funded by NIH grant 1S10OD023481 (to A.M.G.). The tandem mass spectrometry analysis was supported by the UPMC Hillman Cancer Center Proteomics Facility, supported in part by award P30CA047904. This work was supported by NIH grant P50AI150481 (to A.M.G.).

\section{Author contributions}

A.M.G. conceived and directed this study. C.H.B., J.J., M.D. and Y.W. prepared proteins. I.L.B., J.J. and C.H.B. conducted NMR experiments. I.L.B. analyzed NMR data and was responsible for the completion of the NMR/NOE assignments and the NMR structure determination of the $\mathrm{Vpr}_{1-79}-\mathrm{L}-\mathrm{hHR} 23 \mathrm{~A}_{223-363}$ complex. Y.W. carried out crystallization experiments. S.W. carried out X-ray data collection and processing. G.C. performed structure refinement of the X-ray structure. G.C. and I.L.B. analyzed the NMR and X-ray structures and led in providing structural insight. G.C. and J.A. designed and supervised the biochemical experiments. Y.W. carried out mutagenesis and GST-pull down 
experiments. M.D. and Y.W. performed analytical gel-filtration column chromatography. X.Z. carried out ITC experiments. J.S. designed and supervised Vpr-XPC interaction studies, and C.H. carried out transfections, immunoprecipitations and immunoblotting. I.L.B. and G.C. prepared the initial draft of the manuscript and J.S. and A.M.G. led the final manuscript preparation. All authors reviewed, commented on, and approved the manuscript.

\section{Competing interests}

The authors declare no competing interests.

\section{Additional information}

Supplementary information The online version contains supplementary material available at https://doi.org/10.1038/s41467-021-27009-w.

Correspondence and requests for materials should be addressed to Guillermo Calero or Angela M. Gronenborn.

Peer review information Nature Communications thanks the anonymous reviewers for their contribution to the peer review of this work.
Reprints and permission information is available at http://www.nature.com/reprints

Publisher's note Springer Nature remains neutral with regard to jurisdictional claims in published maps and institutional affiliations.

\section{(c) (i)}

Open Access This article is licensed under a Creative Commons Attribution 4.0 International License, which permits use, sharing, adaptation, distribution and reproduction in any medium or format, as long as you give appropriate credit to the original author(s) and the source, provide a link to the Creative Commons license, and indicate if changes were made. The images or other third party material in this article are included in the article's Creative Commons license, unless indicated otherwise in a credit line to the material. If material is not included in the article's Creative Commons license and your intended use is not permitted by statutory regulation or exceeds the permitted use, you will need to obtain permission directly from the copyright holder. To view a copy of this license, visit http://creativecommons.org/ licenses/by/4.0/.

(C) The Author(s) 2021 